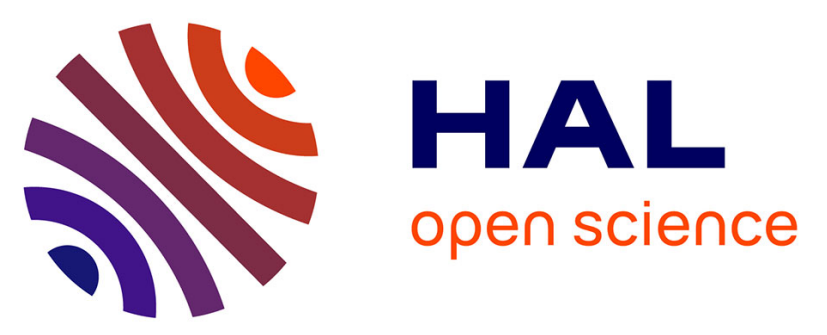

\title{
APPORT DE LA DIFFUSION RAYLEIGH DÉPOLARISÉE ET DE L'EFFET KERR OPTIQUE A L'ÉTUDE DES CORRÉLATIONS RADIALES ET D'ORIENTATION DANS QUELQUES LIQUIDES
}

S. Kielich, J. Lalanne, F. Martin

\section{To cite this version:}

S. Kielich, J. Lalanne, F. Martin. APPORT DE LA DIFFUSION RAYLEIGH DÉPOLARISÉE ET DE L'EFFET KERR OPTIQUE A L'ÉTUDE DES CORRÉLATIONS RADIALES ET D'ORIENTATION DANS QUELQUES LIQUIDES. Journal de Physique Colloques, 1972, 33 (C1), pp.C1-191-C1-205. 10.1051/jphyscol:1972135 . jpa-00214925

HAL Id: jpa-00214925

https://hal.science/jpa-00214925

Submitted on 1 Jan 1972

HAL is a multi-disciplinary open access archive for the deposit and dissemination of scientific research documents, whether they are published or not. The documents may come from teaching and research institutions in France or abroad, or from public or private research centers.
L'archive ouverte pluridisciplinaire HAL, est destinée au dépôt et à la diffusion de documents scientifiques de niveau recherche, publiés ou non, émanant des établissements d'enseignement et de recherche français ou étrangers, des laboratoires publics ou privés. 


\title{
APPORT DE LA DIFFUSION RAYLEIGH DÉPOLARISÉE ET DE L'EFFET KERR OPTIQUE A L'ÉTUDE DES CORRÉLATIONS RADIALES ET D'ORIENTATION DANS QUELQUES LIQUIDES
}

\author{
S. KIELICH (*), J. R. LALANNE et F. B. MARTIN \\ Université de Bordeaux 1 et Centre de Recherches P. Pascal \\ Domaine Universitaire, 33, Talence
}

\begin{abstract}
Résumé. - La diffusion Rayleigh dépolarisée et l'effet Kerr optique s'expriment simplement à partir de l'anisotropie optique effective. Dans le cas des liquides, celle-ci se compose de trois termes traduisant successivement : l'anisotropie moléculaire affectée des corrélations angulaires ; l'effet croisé corrélations angulaires-redistribution ; et la redistribution. Nous montrons comment l'anisotropie effective dépend des corrélations angulaires fortes calculées à partir des énergies d'interaction moléculaire multipolaires. Les résultats expérimentaux concernant les corrélations angulaires existant dans le benzène et le nitrobenzène, et leurs variations thermiques, sont reportés. L'accord entre ces résultats et les prévisions théoriques est satisfaisant, dans l'hypothèse des configurations moléculaires à tendance antiparallèle (nitrobenzène) et perpendiculaire (benzène). Ce travail est une contribution aux études des propriétés optique et électrique des molécules et de l'ordre moléculaire à courte distance régnant dans les liquides.
\end{abstract}

\begin{abstract}
Depolarized Rayleigh scattering and Optical Kerr Effect can be simply described from the effective optical anisotropy. In the case of liquids, this effective anisotropy is composed of three terms : the intrinsic molecular anisotropy corrected by the angular correlations factor; the mixed effect coming from the angular correlations and the redistribution; and the pure redistribution phenomenon. We show how the effective optical anisotropy is related to the strong angular correlations calculated from molecular multipolar interaction energies. Experimental data concerning the angular correlations of benzene and nitrobenzene, and their thermal variations, is reported. Good agreement between these experimental results and theoretical considerations can be found, with the use of simple models, based upon the molecular tendency to antiparallel configuration (nitrobenzene) and quite perpendicular one (benzene). These techniques can give informations about the optical and electrical properties of molecules as well as about the short range order within the liquids.
\end{abstract}

\begin{abstract}
A. Introduction. - Au cours des cinq dernières années, de nombreux travaux, expérimentaux et théoriques [1]-[14], ont été publiés dans le but de préciser les interactions moléculaires radiales et d'orientation dans les liquides et les solutions. Certains résultats obtenus sont en bon accord avec ceux déduits des études d'effet Kerr optique en fonction de la température [15]-[17]. L'étude parallèle de l'effet Kerr optique et de la diffusion Rayleigh dépolarisée permet d'obtenir non seulement des informations sur les polarisabilités linéaire et non linéaire d'ordre 1,2 et 3 [18]-[20] et leurs anisotropies, mais également sur les corrélations radiales et d'orientation existant dans les liquides.
\end{abstract}

Nous donnons ici une analyse détaillée des différents processus microscopiques intervenant dans la diffusion anisotrope de la lumière et dans l'effet Kerr optique

(*) Directeur de l'Institut de Physique de l'Université de Poznan (Pologne), Professeur Associé à l'Université de Bordeaux 1. en mettant, dans chaque cas, l'accent sur les contributions les plus importantes. Nous étudierons ainsi les corrélations d'orientation qui s'avéreront particulièrement fortes dans les liquides constitués de molécules très anisotropes, tels le benzène et le nitrobenzène.

Dans ce cas, un rôle important échoit, non seulement aux corrélations purement orientationnelles, mais aussi à un processus mixte résultant de la coopération entre interactions d'orientation et redistribution spatiale des molécules. L'apport de ce processus peut être soit négatif, soit positif, suivant le signe de l'anisotropie optique et la tendance des molécules au paratropisme (configuration moyenne parallèle) ou au diatropisme (configuration moyenne perpendiculaire).

Dans le cas des molécules isotropes, nous discutons aussi le rôle joué par la redistribution en tenant compte, non seulement des corrélations radiales entre deux molécules, mais aussi des corrélations radiales entre trois ou plusieurs molécules. Ces corrélations peuvent aux hautes densités, devenir importantes et augmenter 
ou abaisser l'anisotropie induite par l'environnement moléculaire.

Enfin, nous rapportons également les résultats de nos mesures de diffusion Rayleigh dépolarisée et d'effet Kerr optique et montrons comment elles permettent l'étude des corrélations angulaires et radiales dans quelques liquides et de leur évolution au cours d'une variation thermique.

B. Considérations théoriques. - I. LA COMPOSANTE DÉPOLARISÉE DE LA DIFFUSION RAYLEIGH. - Généralement, on distingue dans les liquides deux types de diffusion : la diffusion isotrope et la diffusion anisotrope. Les mécanismes microscopiques initiateurs de ces deux processus sont différents et fournissent des renseignements complémentaires sur les molécules et la structure du milieu diffusant. La diffusion isotrope est principalement due aux fuctuations de la densité moléculaire et ne peut que refléter faiblement les interactions moléculaires. Ce type de diffusion est présent dans toutes les substances, quels que soient leur structure et leur état (température du zéro absolu exclue). De ce fait, elle apparaît dans les mesures de la constante de Rayleigh et du rapport de dépolarisation tout comme la diffusion anisotrope, bien plus riche en renseignements structuraux. C'est en effet la diffusion anisotrope qui traduit le mieux la structure moléculaire du milieu diffusant. Dans les substances denses constituées d'atomes ou de molécules à polarisation isotrope, elle est uniquement due aux fluctuations statistiques des champs moléculaires. Les mesures directes de la seule composante anisotrope de la lumière diffusée, selon la méthode de Bothorel [21], semblent, pour cette raison, présenter un intérêt certain en optique moléculaire. Rappelons que nous mesurons la composante anisotrope de la lumière diffusée dont les oscillations sont situées dans le plan d'observation (composante horizontale d'intensité $H$ ), en ayant recours à une onde lumineuse incidente (laser à gaz ou déclenché), se propageant horizontalement, polarisée ou non polarisée. Dans le cas où la lumière incidente est polarisée verticalement, la théorie moléculaire statistique de la diffusion fournit le résultat suivant, valable pour une observation horizontale perpendiculaire au faisceau incident :

$$
H_{V}=\frac{3}{5}\left(\frac{2 \pi}{\lambda_{R}}\right)^{4}\left(\frac{n_{R}^{2}+2}{3}\right)^{2} F_{\text {ani }}\left(\lambda_{R}\right) I
$$

$n_{R}$ est l'indice du milieu diffusant à la longueur d'onde $\lambda_{R}$.

$I$ est l'intensité du faisceau diffusant.

$F_{\text {ani }}\left(\lambda_{R}\right)$ traduit le mécanisme initiateur de la diffusion anisotrope au niveau moléculaire et est défini par :

$F_{\text {ani }}\left(\lambda_{R}\right)=\frac{1}{6 V}\left\langle\sum_{p=1}^{N} \sum_{q=1}^{N} D_{i j\left(\lambda_{R}\right)}^{(p)} D_{i j\left(\lambda_{R}\right)}^{*(q)} \mathrm{e}^{-i s, \mathrm{r}_{p q}}\right\rangle$

$V$ est le volume diffusant contenant $N$ molécules; le symbole $<>$ désigne la moyenne statistique en présence des corrélations moléculaires; la grandeur s. $\mathbf{r}_{p q}$ décrit la différence de phase existant entre la lumière diffusée par la molécule $p$ au point $\mathbf{r}_{p}$ et celle diffusée par la molécule $q$ au point $\mathbf{r}_{q} ; \mathbf{r}_{p q}=\mathbf{r}_{q}-\mathbf{r}_{p}$ est le vecteur reliant les centres des molécules $p$ et $q$; pour la diffusion Rayleigh classique (sans changement de longueur d'onde) on a :

$$
|\mathbf{s}|=\left|\mathbf{k}-\mathbf{k}_{\mathrm{d}}\right|=\frac{4 \pi}{\lambda_{R}} \sin \frac{\vartheta}{2}
$$

$\vartheta$ étant l'angle de diffusion c'est-à-dire l'angle fait par le vecteur d'onde $\mathbf{k}$ de l'onde incidente et celui, $\mathbf{k}_{\mathbf{d}}$, de l'onde diffusée.

Nous avons introduit dans l'équation (2) un tenseur anisotrope de polarisabilité optique, nommé déviateur et défini par:

$$
D_{i j}^{(p)}\left(\lambda_{R}\right)=\frac{1}{2}\left(\frac{\partial m_{i}^{(p)}}{\partial E_{j}^{\lambda_{R}}}+\frac{\partial m_{j}^{(p)}}{\partial E_{i}^{\lambda_{R}}}\right)-\frac{1}{3} \delta_{i j}\left(\frac{\partial m_{k}^{(p)}}{\partial E_{k}^{\lambda_{R}}}\right)
$$

$\mathbf{m}^{(p)}$ est le moment électrique dipolaire induit dans la molécule $p$ par le champ électrique $\mathbf{E}^{\lambda_{R}}$. L'onde incidente a pour longueur $\lambda_{R} . \delta_{i j}$ est le tenseur-unité de Kronecker. Dans les expressions (2) et (4) nous avons utilisé la convention de sommation d'Einstein.

La constante de diffusion anisotrope (2) peut aussi être déterminée à partir des mesures du facteur de dépolarisation $D$ et de la partie isotrope de la constante de Rayleigh $R_{\text {is }}$, par la formule [22] :

$$
F_{\mathrm{ani}}\left(\lambda_{R}\right)=10\left(\frac{\lambda_{R}}{2 \pi}\right)^{4}\left(\frac{3}{n_{\lambda_{R}}^{2}+2}\right)^{2} \frac{R_{\mathrm{is}} D}{6-7 D} .
$$

Ce procédé de détermination de la diffusion anisotrope suppose effectuées les mesures de l'intensité isotrope d'une part, et du facteur de dépolarisation d'autre part. Son intérêt en est par là limité.

II. LA CONSTANTE D'EFFET KERR OPTIQUe. - L'effet Kerr optique, découvert en 1964 par Mayer [23] élargit les possibilités d'étude des microstructures liquides autrefois principalement tributaires des études de diffusion Rayleigh. L'utilisation [24] d'un faisceau d'analyse de longueur d'onde $\lambda_{A}$ (p. ex. un laser à gaz) et d'un faisceau intense inducteur de longueur d'onde $\lambda_{I}$ (par exemple un laser à rubis ou à néodyme) conduit à l'expression. suivante traduisant la différence des indices pour les oscillations parallèle et perpendiculaire à la direction des oscillations du faisceau inducteur d'intensité $I_{L}$ :

$$
n_{\|}\left(\lambda_{A}\right)-n_{\perp}\left(\lambda_{A}\right)=\lambda_{A} B\left(\lambda_{A}, \lambda_{I}\right) I_{L} .
$$

La constante expérimentale $B\left(\lambda_{A}, \lambda_{I}\right)$ d'effet Kerr optique de la formule (6), est liée à la constante $B_{\mathrm{m}}\left(\lambda_{A}, \lambda_{I}\right)$ traduisant les mécanismes moléculaires initiateurs par [25] :

$$
B\left(\lambda_{A}, \lambda_{\mathrm{I}}\right)=\frac{3}{2 \lambda_{A} n_{A}}\left(\frac{n_{\lambda_{A}}^{2}+2}{3}\right)^{2}\left(\frac{n_{\lambda_{\mathrm{I}}}^{2}+2}{3}\right)^{2} B_{\mathrm{m}}\left(\lambda_{A}, \lambda_{I}\right)
$$

$n_{\lambda_{A}}$ et $n_{\lambda_{I}}$ sont les indices de réfraction mesurés aux longueurs d'onde $\lambda_{A}$ et $\lambda_{I}$. 
Dans le cas général, $B_{\mathrm{m}}\left(\lambda_{A}, \lambda_{I}\right)$ se met sous la forme :

$$
B_{\mathrm{m}}\left(\lambda_{A}, \lambda_{I}\right)=B_{\mathrm{m}}^{\mathrm{NL}}\left(\lambda_{A}, \lambda_{I}\right)+B_{\mathrm{m}}^{\mathrm{SF}}\left(\lambda_{A}, \lambda_{I}\right)
$$

où la constante $B_{\mathrm{m}}^{\mathrm{NL}}$ décrit le processus de déformation traduit par la polarisabilité non linéaire du nuage électronique des atomes ou des molécules. La polarisabilité du microsystème subit une variation non linéaire, proportionnelle au carré du champ électrique donc, dans le cas optique, à l'intensité $I_{L}$. C'est l'effet Voigt [26], indépendant de la température, et ne dépendant que faiblement des interactions moléculaires. Son rôle est plus marqué dans les substances atomiques ou moléculaires à l'état gazeux [18]. Dans le cas général, on peut écrire [27] :

$$
\begin{aligned}
& \dot{B}_{\mathrm{m}}^{\mathrm{NL}}\left(\lambda_{A}, \lambda_{I}\right)=\frac{\pi}{45 V}\left\langle\sum _ { p = 1 } ^ { N } \left\{ 3 \frac{\partial^{3} m_{i}^{(p)}}{\partial E_{j}^{\lambda_{A}} \partial E_{i}^{\lambda_{I}} \partial E_{j}^{\lambda_{I}}}+\right.\right. \\
& \left.\left.\quad+3 \frac{\partial^{3} m_{i}^{(p)}}{\partial E_{j}^{\lambda_{A}} \partial E_{j}^{\lambda_{I}} \partial E_{i}^{\lambda_{I}}}-2 \frac{\partial^{3} m_{i}^{(p)}}{\partial E_{i}^{\lambda_{A}} \partial E_{j}^{\lambda_{I}} \partial E_{j}^{\lambda_{I}}}\right\}\right\rangle
\end{aligned}
$$

En première approximation, on pourra négliger les champs moléculaires et écrire le développement en série suivant [28] :

$m_{i}^{(p)}=\left[a_{i j}^{(p)}+b_{i j k}^{(p)} E_{k}^{\lambda_{I}}+\frac{1}{2} c_{i j k l}^{(p)} E_{k}^{\lambda_{I}} E_{l}^{\lambda_{I}}+\cdots\right] E_{j}^{\lambda_{A}}$

où $a_{i j}^{(p)}\left(\lambda_{A}\right)$ est le tenseur de polarisabilité linéaire de la molécule $p$, à la longueur d'onde $\lambda_{A}$; les tenseurs $b_{i j k}^{(p)}\left(\lambda_{A}, \lambda_{I}\right)$ et $c_{i j k l}^{(p)}\left(\lambda_{A}, \lambda_{I}\right)$ sont ceux des polarisabilités non linéaires d'ordres 2 et 3 , induites dans la molécule $p$ par le faisceau inducteur intense [28], [29].

En substituant (10) dans l'éq. (9), on obtient [28] :

$B_{\mathrm{m}}^{\mathrm{NL}}\left(\lambda_{A}, \lambda_{I}\right)=\frac{\pi \rho}{45}\left(3 c_{i j i j}^{\lambda_{A}, \lambda_{I}}+3 c_{i j j i}^{\lambda_{A}, \lambda_{I}}-2 c_{i i j j}^{\lambda_{A}, \lambda_{I}}\right)$

$\rho=N / V$ étant le nombre de molécules par unité de volume.

La deuxième constante $B_{\mathrm{m}}^{\mathrm{SF}}$ de la formule (8) résulte de différents processus moléculaires statistiques dont le plus important, dans le cas des molécules naturellement anisotropes, est celui de la réorientation moléculaire de Langevin [30]. Un autre processus, de grande importance dans les substances atomiques ou constituées de molécules optiquement isotropes, est celui traduisant les fluctuations de translation d'Yvon [31] et Kirkwood [32]. Celles-ci, en favorisant des regroupements ou réarrangements spatiaux induisent une anisotropie apparente du milieu [22], [23].

Nous venons de mentionner deux processus très différents. L'un se produit dans le cas des molécules naturellement anisotropes à l'état fondamental. L'autre traduit l'anisotropie induite au sein de groupements d'atomes ou de molécules isotropes en corrélation. Le calcul laisse supposer l'existence de plusieurs effets (7), (34) que nous appellerons croisés, et qui seront décrits par la suite. L'expression la plus générale de $B_{\mathrm{m}}^{\mathrm{SF}}$ s'écrit (27) :

$$
B_{\mathrm{m}}^{\mathrm{SF}}\left(\lambda_{A}, \lambda_{I}\right)=\frac{2 \pi}{15 V k T}\left\langle\sum_{p=1}^{N} \sum_{q=1}^{N} D_{i j}^{(p)}\left(\lambda_{A}\right) D_{i j}^{(q)}\left(\lambda_{I}\right)\right\rangle
$$

où $D_{i j}\left(\lambda_{A}\right)$ et $D_{i j}\left(\lambda_{I}\right)$ sont les déviateurs de la polarisabilité d'ordre un, définis comme le tenseur (4). En négligeant les interférences en diffusion Rayleigh dépolarisée $\left(\mathrm{sr}_{p q} \ll 1\right)$ et en supposant

$$
\lambda_{A} \# \lambda_{I} \# \lambda_{R}=\lambda
$$

on montre que les constantes $F_{\text {ani }}(\lambda)$ et $B_{\mathrm{m}}^{\mathrm{SF}}(\lambda, \lambda)$, définies par les équations (2) et (12), s'expriment simplement l'une par rapport à l'autre. Dans ce cas, les équations (2) et (12) conduisant à la relation suivante [34] :

$$
B_{\mathrm{m}}^{\mathrm{SF}}(\lambda)=\frac{4 \pi}{5 k T} F_{\mathrm{ani}}(\lambda)=\frac{4 \pi \rho}{45 \frac{k T}{k}} \Gamma_{\lambda}^{2}
$$

$\Gamma_{\lambda}^{2}$ est l'anisotropie moléculaire effective définie par :

$$
\Gamma_{\lambda}^{2}=\frac{3}{2 N}\left\langle\sum_{p=1}^{N} \sum_{q=1}^{N} D_{i j}^{(p)}(\lambda) D_{i j}^{(q)}(\lambda)\right\rangle .
$$

Cette grandeur caractérise la partie dépendante de la température de la constante d'effet Kerr optique et la diffusion anisotrope de la lumière par les liquides constitués de molécules dont les dimensions linéaires et les distances mutuelles de corrélation sont petites par rapport à la longueur d'onde de la lumière. Dans ces conditions, et en absence d'absorption optique, le tenseur de déviation est réel et hermitien : $D_{i j}=D_{j i}^{*}$.

C. Evaluation de l'anisotropie optique effective. Afin de pouvoir utiliser le paramètre d'anisotropie optique $\Gamma_{\lambda}^{2}$, il faut supposer certaines hypothèses et faire quelques approximations que nous allons maintenant rappeler. Considérons en particulier le calcul des valeurs moyennes définies en mécanique statistique par :

$$
<G>=\frac{\int G\left(\tau^{N}\right) \exp \left\{-\frac{U\left(\tau^{N}\right)}{k T}\right\} \mathrm{d} \tau^{N}}{\int \exp \left\{-\frac{U \tau^{N}}{k T}\right\} \mathrm{d} \tau^{N}}
$$

où $\tau^{N}$ est l'ensemble des variables définissant la configuration des $N$ molécules du milieu (généralement, ce seront les variables de position $\mathbf{r}^{N}=\mathbf{r}_{1}, \mathbf{r}_{2}, \ldots, \mathbf{r}_{N}$ et d'orientation $\Omega^{N}=\Omega_{1}, \Omega_{2}, \ldots, \Omega_{N}$ ).

L'énergie potentielle totale $U\left(\tau^{N}\right)$ d'interaction des $N$ molécules sera séparée en une partie centrale $U_{0}\left(r^{N}\right)$, ne dépendant que des positions des molécules, et en une partie non centrale $W\left(r^{N}, \Omega^{N}\right)$ dépendant, en outre, de leurs orientations. Généralement, l'énergie d'interaction d'orientation, $W$, se traduit par un tenseur d'expression compliquée si l'on tient compte 
des interactions multipolaires [35]. Les calculs statistiques numériques de l'anisotropie (14) ne sont alors possibles que si l'on applique les procédés du calcul classique des perturbations à la valeur moyenne (15). On fera l'hypothèse d'une énergie $W$, petite devant $k T$, et traitée comme petite perturbation à $U_{0}$. Montrons que, dans ce cas, l'approximation d'ordre $0(W=0)$ conduit à une anisotropie effective (15) se réduisant à l'anisotropie de la molécule isolée. Exprimons pour cela le tenseur de déviation $D_{i j}$ dans le référentiel (indices $\alpha, \beta, \ldots$ ) moléculaire principal. Il vient :

$$
D_{i j}^{(p)}=c_{i \alpha}^{(p)} c_{j \beta}^{(p)} D_{\alpha \beta}^{(p)}
$$

$c_{i \alpha}$ est le cosinus directeur de l'angle fait par les axes $i$ et $\alpha$ des deux référentiels. En introduisant (16) dans l'expression (14) de l'anisotropie, il vient :

$$
\Gamma_{\lambda}^{2}=\frac{3}{2 N}\left\langle\sum_{p=1}^{N} \sum_{q=1}^{N} D_{\alpha \beta}^{(p)} D_{\gamma \delta}^{(q)} c_{\alpha \gamma}^{(p q)} c_{\beta \delta}^{(p q)}\right\rangle
$$

$c_{\alpha \gamma}^{(p q)}=c_{i \alpha}^{(p)} c_{i \gamma}^{(q)}$ est le cosinus de l'angle formé par l'axe $\alpha$ de la molécule $p$ et celui $\gamma$ de la molécule $q$. Compte tenu du développement (10), et dans l'approximation linéaire, le déviateur (4) prend la forme :

$$
D_{\alpha \beta}^{(p)}=a_{\alpha \beta}^{(p)}-a_{p} \delta_{\alpha \beta}
$$

où

$$
a=\frac{a_{\alpha x}}{3}=\frac{a_{11}+a_{22}+a_{33}}{3}
$$

est la polarisabilité moyenne de la molécule isolée. Dans un référentiel moléculaire donné, et en absence d'énergie d'orientation, le déviateur est constant et on pourra écrire l'expression (17) sous la forme :

$$
\begin{aligned}
\Gamma_{\lambda}^{2}=\frac{3}{2} D_{\alpha \beta}^{\lambda} D_{\gamma \dot{\delta}}^{\lambda}\left\langle\sum_{p=1}^{N} \sum_{q=1}^{N} c_{\alpha \gamma}^{(p q)} c_{\beta \delta}^{(p q)}\right\rangle_{0} \\
=\frac{3}{2 N} D_{\alpha \beta}^{\lambda} D_{\gamma \delta}^{\lambda}\left\{\sum_{p=1}^{N}\left\langle c_{\alpha \gamma}^{(p p)} c_{\beta \delta}^{(p p)}\right\rangle_{0}+\right. \\
\left.+\sum_{p=1}^{N} \sum_{(q \neq p)}^{N}\left\langle c_{\alpha \gamma}^{(p q)} c_{\beta \delta}^{(p q)}\right\rangle_{0}\right\} .
\end{aligned}
$$

Mais puisque

$$
c_{\alpha \gamma}^{(p p)}=\delta_{\alpha \gamma} \quad \text { et }\left\langle c_{\alpha \gamma}^{(p q)} c_{\beta \delta}^{(p q)}>_{0}=\frac{\delta_{\alpha \beta} \delta_{\gamma \delta}}{3}\right.
$$

et en tenant compte de la relation (18), on obtient finalement :

$$
\Gamma_{\lambda}^{2}=\frac{3}{2} D_{\alpha \beta}^{\lambda} D_{\alpha \beta}^{\lambda}=\frac{1}{2}\left(3 a_{\alpha \beta}^{\lambda} a_{\alpha \beta}^{\lambda}-a_{\alpha \alpha}^{\lambda} a_{\beta \beta}^{\lambda}\right) .
$$

c'est-à-dire l'anisotropie de la molécule isolée.

Les approximations que nous. venons de décrire permettent le calcul de l'anisotropie effective dans le cas où les corrélations d'orientation sont faibles. Des résultats, appliqués à l'ensemble des symétries moléculaires, ont été publiés [2], [7].
Une situation bien différente se présente dans les liquides constitués de molécules très anisotropes. Les corrélations d'orientation sont alors très marquées et le calcul de perturbation n'est plus possible. On voit alors que l'obtention de résultats numériques concrets nécessite, dans ce cas, le recours à des modèles structuraux très simples. Ces deux cas, très différents, seront étudiés séparément.

1. INFLUENCE DES CORRÉLATIONS ANGULAIRES : CAS DES LIQUIDES A MOLÉCULES ANISOTROPES. - Revenons au tenseur de déviation sous la forme (16) qui, dans l'approximation (18), peut s'écrire de la manière suivante :

$$
D_{i j}^{(p)}=c_{i \alpha}^{(p)} c_{j \beta}^{(p)} a_{\alpha \beta}-a \delta_{i j}
$$

ou en effectuant le changement de référentiel conduisant aux axes principaux de la molécule,

$$
\begin{aligned}
D_{i j}^{(p)}= & \frac{1}{2}\left(a_{11}-a_{22}\right)\left(c_{i 1}^{(p)} c_{j 1}^{(p)}-c_{i 2}^{(p)} c_{j 2}^{(p)}\right)+ \\
& +\frac{1}{2}\left(2 a_{33}-a_{11}-a_{22}\right)\left(c_{i 3}^{(p)} c_{j 3}^{(p)}-\frac{1}{3} \delta_{i j}\right)
\end{aligned}
$$

$a_{11}, a_{22}$ et $a_{33}$ étant les éléments du tenseur de polarisabilité selon les axes principaux (polarisabilités principales).

En substituant ce tenseur de déviation dans la formule (14) de l'anisotropie effective, on obtient :

$$
\Gamma_{\lambda}^{2}=\gamma_{\lambda}^{2}\left(1+J_{11}^{A}+J_{13}^{A}+J_{33}^{A}\right)=\gamma_{\lambda}^{2}\left(1+J_{A}\right)
$$

où

$$
\begin{array}{r}
\gamma_{\lambda}^{2}=\frac{1}{2}\left\{\left(a_{11}^{\lambda}-a_{22}^{\lambda}\right)^{2}+\left(a_{22}^{\lambda}-a_{33}^{\lambda}\right)^{2}+\right. \\
\left.+\left(a_{33}^{\lambda}-a_{11}^{\lambda}\right)^{2}\right\}
\end{array}
$$

est l'anisotropie optique de la molécule isolée donnée par la formule (19), tandis que les grandeurs rendant compte des corrélations moléculaires angulaires sont définies par :

$$
\begin{gathered}
J_{11}^{A}=\frac{3 Z}{8 \gamma_{\lambda}^{2}}\left(a_{11}^{\lambda}-a_{22}^{\lambda}\right)^{2} \times \\
\times<\cos ^{2} \theta_{11}^{(p q)}-\cos ^{2} \theta_{12}^{(p q)}-\cos ^{2} \theta_{21}^{(p q)}+\cos ^{2} \theta_{22}^{(p q)}>
\end{gathered}
$$

$$
J_{13}^{A}=\frac{3 Z}{8 \gamma_{\lambda}^{2}}\left(a_{11}^{\lambda}-a_{22}^{\lambda}\right)\left(2 a_{33}^{\lambda}-a_{11}^{\lambda}-a_{22}^{\lambda}\right) \times
$$

$x<\cos ^{2} \theta_{13}^{(p q)}+\cos ^{2} \theta_{31}^{(p q)}-\cos ^{2} \theta_{23}^{(p q)}-\cos ^{2} \theta_{32}^{(p q)}>$

$$
J_{33}^{A}=\frac{3 Z}{8 \gamma_{\lambda}^{2}}\left(2 a_{33}^{\lambda}-a_{11}^{\lambda}-a_{22}^{\lambda}\right)^{2}\left\langle\cos ^{2} \theta_{33}^{(p q)}-\frac{1}{3}\right\rangle
$$

$z=N-1$ est le nombre de voisins de la molécule $p$ tandis que $\theta_{11}^{(p q)}, \ldots$ sont les angles formés par les axes principaux de la molécule $p$ avec ceux de sa voisine $q$. 
a) Molécules de Langevin. - Considérons d'abord le cas de molécules de révolution autour de l'axe 3. Il vient : $a_{11}=a_{22} \neq a_{33}$, et l'anisotropie $(19 a)$ se réduit à :

$$
\gamma_{\lambda}^{2}=\left(a_{33}^{\lambda}-a_{11}^{\lambda}\right)^{2}
$$

les paramètres des corrélations angulaires (21) et (22) s'annulent; d'autre part, (23) se réduit à la forme bien connue [36], [37] :

$$
J_{A}=J_{33}^{A}=\frac{1}{2} Z\left\{3<\cos ^{2} \theta_{p q}>-1\right\} .
$$

Calculons ce paramètre dans le cas du benzène au sein duquel nous supposerons exister des corrélations angulaires fortes. Le cas théorique des corrélations faibles a déjà été traité [2]. Afin de pouvoir calculer effectivement la moyenne statistique $\left\langle\cos ^{2} \theta_{p q}\right\rangle$, définie en général par la formule (15), il nous faut exprimer l'énergie potentielle $U\left(\tau^{N}\right)$ en fonction de l'angle $\theta_{p q}$. Ce procédé a déjà été employé par un certain nombre d'auteurs dans la théorie de la saturation diélectrique des liquides [38]-[40]. On y parvient (voir Appendice A) en choisissant un modèle, volontairement très simplifié, dans lequel les axes sénaires de deux molécules en interaction restent toujours dans un même plan. Avec ce modèle, l'énergie $U(\tau)$ est une fonction paire de $\cos \theta_{p q}$ et dépend aussi de la distance $r_{p q}$ entre les molécules $p$ et $q$. Afin d'éviter une intégration sur les distances intermoléculaires, nous supposerons que les molécules se trouvent séparées par une distance déterminée à partir de la relation :

$$
r_{p q}=\left(\frac{6 u}{\pi \rho}\right)^{1 / 3}=\left(\frac{g}{\rho}\right)^{1 / 3}
$$

où $\rho$ désigne le nombre de molécules par unité de volume. $u$ est un paramètre numérique dont la valeur, pour les liquides, varie entre 0,6 et 0,74 [41]. On peut alors écrire (voir Appendice) :

$$
-\frac{U_{N}}{k T}=y_{0}-y_{2} \cos ^{2} \theta_{p q}+y_{4} \cos ^{4} \theta_{p q}
$$

où $y_{0}$ rend compte de l'énergie radiale, indépendante de l'angle $\theta_{p q}$ et

$$
\begin{aligned}
y_{2}= & \frac{9 \Theta^{2}}{k g^{5 / 3}} \frac{\rho^{5 / 3}}{T}-\frac{3 h v}{8 k g^{2}} \gamma_{0}\left(a_{0}+\gamma_{0}\right) \frac{\rho^{2}}{T}- \\
& -\frac{135 \Theta \Phi}{4 k g^{7 / 3}} \frac{\rho^{7 / 3}}{T}+\frac{3 \Theta^{2}}{4 k g^{8 / 3}}\left(3 a_{0}-4 \gamma_{0}\right) \frac{\rho^{8 / 3}}{T}
\end{aligned}
$$

$$
y_{4}=-\frac{525}{8} \frac{\Theta \Phi}{\mathrm{kg}^{7 / 3}} \frac{\rho^{7 / 3}}{T}+\frac{3 \Theta^{2}}{8 \mathrm{~kg}^{8 / 3}}\left(15 a_{0}+22 \gamma_{0}\right) \frac{\rho^{8 / 3}}{T}
$$

décrivent successivement les énergies en $\cos ^{2} \theta_{p q}$ et $\cos ^{4} \theta_{p q}, a_{0}$ et $\gamma_{0}$ désignent la polarisabilité électrique moyenne et son anisotropie ; $\Theta$ et $\Phi$ sont les moments électriques quadrupolaire et hexadécapolaire. Ainsi, le paramètre $y_{2}$, qui joue le rôle le plus important, se compose des énergies d'interaction quadrupôle-quadrupôle, dipôle induit-dipôle inđuit (interaction dispersionnelle anisotrope), quadrupôle-hexadécapôle, et quadrupôle-dipôle induit.

Compte tenu du développement (26) et de la définition (15), nous écrirons le paramètre de corrélations anglaires (24) comme suit :

$$
J_{A}=\frac{1}{2} Z\left\{3 L_{2}\left(-y_{2}, \pm y_{4}\right)-1\right\}
$$

avec :

$$
\begin{aligned}
\mathrm{L}_{2}\left(-y_{2}, \pm y_{4}\right)= & \frac{\int_{0}^{\pi} \cos ^{2} \theta_{p q} \exp \left\{-y_{2} \cos ^{2} \theta_{p q} \pm y_{4} \cos ^{4} \theta_{p q}\right\} \sin \theta_{p q} \mathrm{~d} \theta_{p q}}{\int_{0}^{\pi} \exp \left\{-y_{2} \cos ^{2} \theta_{p q} \pm y_{4} \cos ^{4} \theta_{p q}\right\} \sin \theta_{p q} \mathrm{~d} \theta_{p q}} \\
& =\frac{\int_{-1}^{+1} t^{2} \exp \left(-y_{2} t^{2} \pm y_{4} t^{4}\right) \mathrm{d} t}{\int_{-1}^{+1} \exp \left(-y_{2} t^{2} \pm y_{4} t^{4}\right) \mathrm{d} t}
\end{aligned}
$$

$\mathrm{L}_{2}$ décrit la fonction de Langevin d'ordre 2. En omettant le paramètre $y_{4}, L_{2}$ s'écrit [42] :

$$
\mathrm{L}_{2}\left( \pm y_{2}\right)=\mp \frac{1}{2 y_{2}} \pm \frac{1}{2 y_{2}^{1 / 2} I\left( \pm y_{2}\right)}
$$

les intégrales

$$
I\left( \pm y_{2}\right)=\exp \left(\mp y_{2}\right) \int_{\theta}^{\sqrt{y_{2}}} \exp \left( \pm t^{2}\right) \mathrm{d} t
$$

ont été calculées numériquement.
La figure 1 montre l'allure des variations du paramètre de corrélations angulaires $J_{A} / Z$ (défini par la formule $24 a$ ) en fonction de $\pm y_{2}$, dans le cas de molécules ayant tendance à une configuration de type parallèle $\left(+y_{2}\right)$ et dans celui de molécules à tendance de type perpendiculaire $\left(-y_{2}\right)$. Dans le cas intéressant des corrélations très fortes, c'est-à-dire celui pour lequel $y_{2} \rightarrow \infty, \mathrm{L}_{2}\left(+y_{2}\right)$ tend vers 1 et $J_{A}$ vers $Z$ tandis que $\mathrm{L}_{2}\left(-y_{2}\right)$ tend vers 0 et $J_{A}$ vers $-Z / 2$. Ce dernier cas correspond à une structure dans laquelle les molécules de benzène sont figées dans des positions 
de type perpendiculaire, voisines de celles existant à l'état solide. On voit que, dans le cas de saturation des corrélations angulaires, le paramètre $J_{A}( \pm \infty)$ donne directement le nombre $Z$ des plus proches voisins.

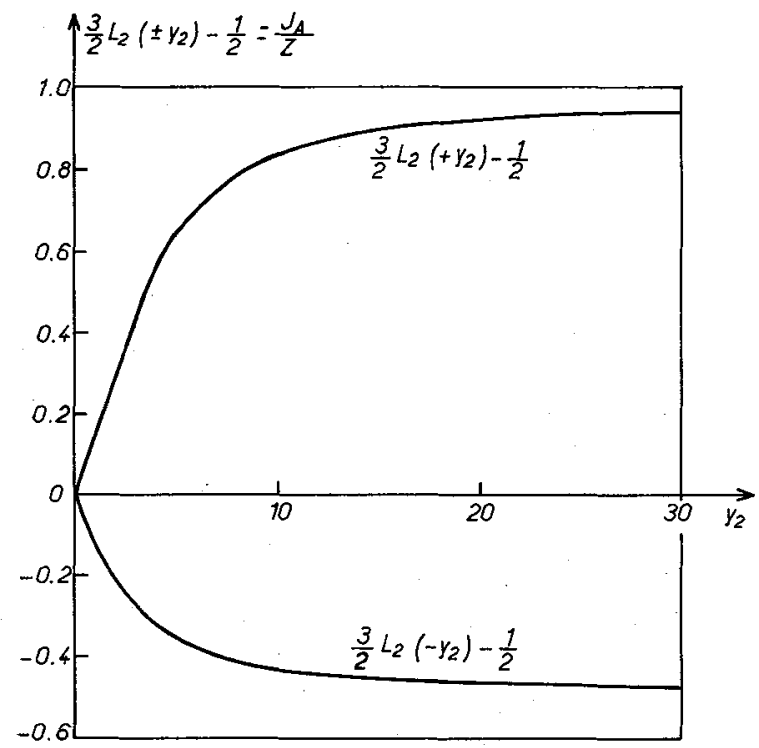

Fig. 1. - Valeur du paramètre $J_{A} / Z$ en fonction de $Y_{2}$.

b) Molécules sans centre de symétrie. - Etudions le cas des molécules dipolaires, dépourvues de symétrie axiale. Pour de telles molécules, les trois composantes (21)-(23) du paramètre de corrélations angulaires sont différentes de zéro. On peut les calculer assez simplement dans l'hypothèse des corrélations angulaires faibles. Cette hypothèse n'est, sans aucun doute, pas vérifiée dans le cas du nitrobenzène, que nous avons étudié et pour lequel nous aurons recours au modèle particulier [39], [40], selon lequel les molécules du nitrobenzène ont tendance à s'orienter en paires de partenaires antiparallèles. Ceci nous permet de réduire l'énergie $U_{N}$ à une seule fonction de l'angle $\theta_{p q}$ $\left(\theta_{p q}\right.$ étant l'angle entre les dipôles électriques $\boldsymbol{\mu}_{p}$ et $\left.\boldsymbol{\mu}_{q}\right)$. L'énergie de telles paires prend la forme suivante (voir Appendice),

$-\frac{U_{N}}{k T}=y_{0}+y_{1} \cos \theta_{p q}-y_{2} \cos ^{2} \theta_{p q}+y_{3} \cos ^{3} \theta_{p q}-\cdots$

où nous avons introduit la notation :

$$
\begin{array}{r}
y_{1}=\frac{\mu^{2}}{k g} \frac{\rho}{T}-\frac{3 \mu \Omega}{k g} \frac{\rho^{5 / 3}}{T}+\frac{15 \Omega^{2}}{4 k g^{7 / 3}} \frac{\rho^{7 / 3}}{T} \\
y_{2}=\frac{3 \Theta^{2}}{2 k g^{5 / 3}} \frac{\rho^{5 / 3}}{T}-\frac{\gamma_{0}}{k g^{2}}\left(\mu^{2}+\frac{1}{8} h v \gamma_{0}\right) \frac{\rho^{2}}{T}- \\
\quad-\frac{15 \Theta \Phi}{2 k g^{7 / 3}} \frac{\rho^{7 / 3}}{T}
\end{array}
$$

$y_{3}=\frac{5 \Omega^{2}}{2 \mathrm{~kg}^{7 / 3}} \frac{\rho^{7 / 3}}{T}$ $\mu, \Theta, \Omega, \Phi$ désignent respectivement les moments électriques dipolaire, quadrupolaire, octopolaire et hexadécapolaire.

L'énergie (31) ne dépendant que de l'angle $\Theta_{p q}$ fait par les directions des dipôles électriques on calcule les paramètres angulaires (21) et (22) en effectuant d'abord la moyenne sur les angles d'Euler azimuthaux. Elle entraîne l'annulation de $J_{11}^{A}$ et $J_{13}^{A}$ :

$$
J_{11}^{A}=0 ; \quad J_{13}^{A}=0 .
$$

Avec l'énergie donnée par la formule (31), l'unique paramètre qui ne s'annule pas est celui donné par la formule (23), que nous écrirons sous la forme suivante :

$$
\begin{aligned}
J_{A} & =J_{33}^{A}=\frac{1}{2} K Z\left\{3<\cos ^{2} \theta_{p q}>-1\right\} \\
& =\frac{1}{2} K Z\left\{3 \mathrm{~L}_{2}\left(y_{1}, \pm y_{2}, \pm y_{3}\right)-1\right\}
\end{aligned}
$$

où l'on a $K$ par :

$K=\frac{\left[2 a_{33}^{\lambda}-a_{11}^{\lambda}-a_{22}^{\lambda}\right]^{2}}{4 \gamma_{\lambda}^{2}}=\mathbb{l}-\frac{3\left(a_{11}^{\lambda}-a_{22}^{\lambda}\right)^{2}}{4 \gamma_{\lambda}^{2}}$

et les fonctions de Langevin d'ordre 2 par :

$$
\begin{aligned}
& \mathrm{L}_{2}\left(y_{1}, \pm y_{2}, \pm y_{3}\right)= \int_{-1}^{+1} t^{2} \exp \left(y_{1} t \pm y_{2} t^{2} \pm y_{3} t^{3}\right) \mathrm{d} t \\
&=\frac{\int_{-1}^{+1} \exp \left(y_{1} t \pm y_{2} t^{2} \pm y_{3} t^{3}\right) \mathrm{d} t}{}
\end{aligned}
$$

En omettant dans l'expression ci-dessus le paramètre $y_{3}$, on obtient [42] :

$$
\begin{aligned}
& \mathbf{L}_{2}\left(y_{1}, \pm y_{2}\right)=\frac{y_{1}^{2} \mp 2 y_{2}}{4 y_{2}^{2}} \pm \\
& \quad \pm \frac{\mathrm{e}^{y_{1}}+\mathrm{e}^{-y_{1}}}{4 y_{2}^{1 / 2} I\left(y_{1}, \pm y_{2}\right)}-\frac{y_{1}\left(\mathrm{e}^{y_{1}}-\mathrm{e}^{-y_{1}}\right)}{8 y_{2}^{3 / 2} I\left(y_{1}, \pm y_{2}\right)}
\end{aligned}
$$

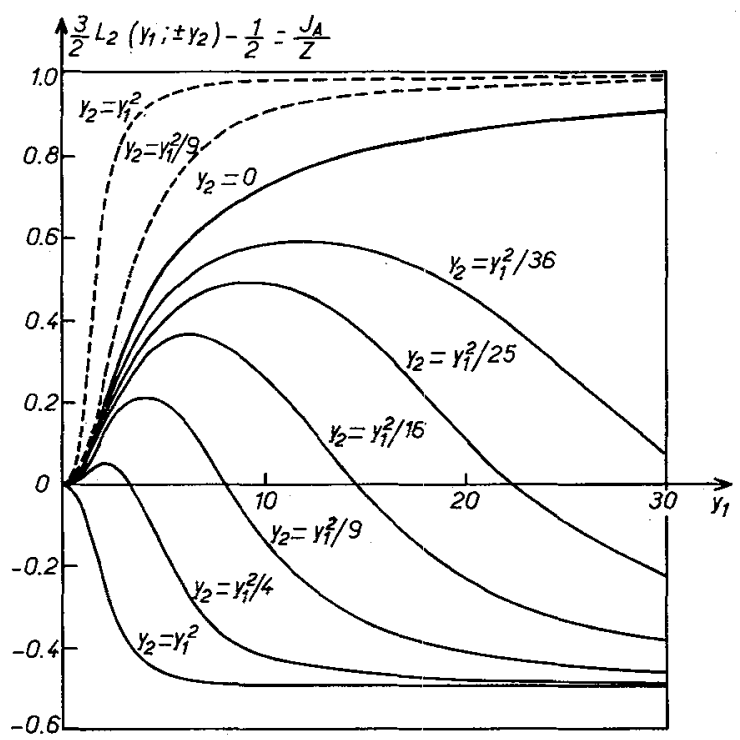

FIG. 2. - Valeur du paramètre $J_{A} / Z$ en fonction de $Y_{1}$, pour différentes valeurs du rapport $Y_{2} / Y_{1}$. En trait plein : $3 / 2 \mathbf{L}_{2}\left(Y_{1}-Y_{2}\right)-1 / 2$. En pointillé : $3 / 2 \mathbf{L}_{2}\left(Y_{1}+Y_{2}\right)-1 / 2$. 
où n'apparaissent que les intégrales calculées numériquement :

$$
\begin{aligned}
I\left(y_{1}, \pm y_{2}\right)= & \frac{1}{2} \exp \left\{\mp\left(\frac{y_{1}^{2}}{4 y_{2}}+y_{2}\right)\right\} \times \\
& \times \int_{-\sqrt{y_{2}} \pm\left(y_{1} / 2 \sqrt{y_{2}}\right)}^{+\sqrt{y_{2}} \pm\left(y_{1} / 2 \sqrt{y_{2}}\right)} \exp \left( \pm t^{2}\right) \mathrm{d} t
\end{aligned}
$$

La figure 2 fournit les courbes traduisant les variations de $J_{A} / Z$ en fonction de $y_{1}$ et $y_{2}$. A la saturation de corrélation $L_{2}(\infty)$ tend vers $1, J_{A}$ tend vers $K Z$ (éq. (34)). La connaissance de $K$ permet alors le calcul du nombre $Z$ de proches voisins.

2. INFLUENCE DES FLUCTUATIONS ANISOTROPES DU CHAMP MOLÉCulaire. - a) Formule générale. - Nous n'avons jusque-là tenu compte que de la polarisabilité linéaire des molécules, polarisabilité due uniquement à l'action du champ électrique $\mathbf{E}^{\lambda}$ de l'onde lumineuse. Généralement, dans les milieux denses, il existe également au niveau de la molécule considérée $p$, un champ électrique interne dû à la distribution de charges créé par les autres molécules du milieu, polarisées par le champ $\mathbf{E}^{\lambda}$. Dans l'approximation dipolaire, ce champ $\mathbf{F}^{(p)}$ est de la forme :

$$
F_{i}^{(p)}=F_{o i}^{(p)}-\sum_{s=1}^{N} T_{i j}^{(p s)} m_{j}^{(s)}
$$

$F_{o i}^{(p)}$ étant le champ moléculaire en absence de l'onde lumineuse, et $T_{i j}^{(p s)}$ le tenseur d'interaction dipôledipôle [31], [32] défini par :

$$
T_{i j}^{(p s)}=-r_{p s}^{-5}\left(3 r_{p s i} r_{p s j}-r_{p s}^{2} \delta_{i j}\right) \text {. }
$$

Nous écrirons donc pour un milieu dense et dans l'approximation linéaire

$$
m_{i}^{(p)}=a_{i j}^{(p)}\left(E_{j}^{\lambda}+F_{j}^{(p)}\right)
$$

expression qui, introduite dans (4), conduit au tenseur de déviation suivant :

$$
\begin{aligned}
D_{i j}^{(p)}(\lambda) & =a_{i j}^{(p)}-a_{j} \delta_{i j}+ \\
+ & \frac{1}{2}\left(a_{i k}^{(p)} \frac{\partial F_{k}^{(p)}}{\partial E_{j}^{\lambda}}+a_{j k}^{(p)} \frac{\partial F_{k}^{(p)}}{\partial E_{i}^{\lambda}}\right)-\frac{1}{3} \delta_{i j}\left(a_{k l}^{(p)} \frac{\partial F_{l}^{(p)}}{\partial E_{k}}\right)
\end{aligned}
$$

ce tenseur contient maintenant une nouvelle contribution due aux fluctuations du champ moléculaire dans le liquide. Cependant, la solution générale de notre problème pose d'assez grandes difficultés, et nous nous bornerons à l'étude de quelques cas simples.

b) Application aux molécules à polarisabilité isotrope. - Les molécules du milieu étant isotropement polarisables, le tenseur de polarisabilité s'écrit :

$$
a_{i j}^{(p)}=a_{p} \delta_{i j}
$$

expression qui, introduite dans (41), donne :

$D_{i j}^{(p)}(\lambda)=\frac{1}{2} a_{p}\left\{\frac{\partial F_{i}^{(p)}}{\partial E_{j}^{\lambda}}+\frac{\partial F_{j}^{(p)}}{\partial E_{i}^{\lambda}}-\frac{2}{3} \delta_{i j}\left(\frac{\partial F_{k}^{(p)}}{\partial E_{k}^{\lambda}}\right)\right\}$.
En absence d'absorption optique à la longueur d'onde $\lambda$, on pourra écrire le déviateur (43) sous la forme :

$$
\begin{aligned}
D_{i j}^{p}(\lambda) & =a_{p} \Delta\left(\frac{\partial F_{i}^{(p)}}{\partial E_{j}^{\lambda}}\right) \\
& =a_{p}\left\{\frac{\partial F_{i}^{(p)}}{\partial E_{j}^{\lambda}}-\left\langle\frac{\partial F_{i}^{(p)}}{\partial E_{j}^{\lambda}}\right\rangle_{\Omega}\right\} .
\end{aligned}
$$

Le symbole $\Delta$ définit les fluctuations anisotropes du champ moléculaire. Le symbole $<>\Omega$ définit la moyenne sur toutes les orientations moléculaires. Nous voyons, d'après ce résultat fondamental que, dans le cas d'atomes et de molécules à polarisabilité isotrope, le déviateur ne diffère de zéro que si le liquide présente des fluctuations spatiales des champs moléculaires. Dans ce cas, l'anisotropie effective (14), est également non nulle et prend la forme :

$\Gamma_{\lambda}^{2}=\frac{3}{2 N}\left\langle\sum_{p=1}^{N} \sum_{q=1}^{N} a_{p} a_{q} A\left(\frac{\partial F_{i}^{(p)}}{\partial E_{j}^{\lambda}}\right) \Delta\left(\frac{\partial F_{i}^{(q)}}{\partial E_{j}^{\lambda}}\right)\right\rangle$.

Nous voyons bien que la diffusion anisotrope et la biréfringence optique dans les liquides constitués d'atomes ou de molécules à polarisabilité isotrope sont dues aux fluctuations anisotropes des champs moléculaires [22], [33]. Dans le langage d'Yvon [31] et Kirkwood [23], il s'agit des fluctuations moléculaires de translation. D'après Hellwarth [14], [43] ce phénomène prend le nom de redistribution spatiale.

Les équations (39) et (40) permettent d'écrire, en première approximation :

$$
F_{i}^{(p)}=F_{o i}^{(p)}-\sum_{s=1}^{N} T_{i k}^{(p s)} a_{k l}^{(s)} E_{l}^{\lambda}
$$

et par conséquent, d'après (42), on obtient pour les molécules à polarisabilité isotrope :

$$
\frac{\partial F_{i}^{(p)}}{\partial E_{j}^{\lambda}}=-\sum_{s=1}^{N} a_{s} T_{i j}^{(p s)}
$$

expression qui, substituée dans (44), donne :

$$
\begin{aligned}
\Gamma_{\lambda}^{2}=\frac{3}{2 N}\left\langle\sum_{p=1}^{N} \sum_{q=1}^{N} \sum_{s=1}^{N} \sum_{t=1}^{N} a_{p} a_{q} a_{s} a_{t} \times\right. \\
\left.\times \Delta T_{i j}^{(p s)} \Delta T_{i j}^{(q t)}\right\rangle .
\end{aligned}
$$

Cette formule, traitée par les méthodes bien connues de la mécanique statistique, donne :

$$
\Gamma_{\lambda}^{2}=9 a_{\lambda}^{4}\left\{J_{R}^{(2)}+2 J_{R}^{(3)}+\cdots\right\}=9 a_{\lambda}^{4} J_{R}
$$

où

$$
\begin{aligned}
J_{R}^{(2)} & =\frac{2 \rho}{V} \iint r_{p q}^{-6} g\left(\mathbf{r}_{p}, \mathbf{r}_{q}\right) \mathrm{d} \mathbf{r}_{p} \cdot \mathrm{d} \mathbf{r}_{q} \\
& =8 \pi \rho \int p_{p q}^{-6} g\left(r_{p q}\right) r_{p q}^{2} \mathrm{~d} r_{p q}
\end{aligned}
$$


$J_{R}^{(2)}$ est le paramètre de corrélations radiales binaires [33], dans lequel intervient la fonction de corrélation binaire. Cette fonction est notée $g\left(r_{p}, r_{q}\right)$ pour les molécules $p$ et $q$ situées aux positions $r_{p}$ et $r_{q}$, et $g\left(r_{p q}\right)$ si, ayant fixé la position de la molécule $p$, on introduit la distance relative $r_{p q}$.

D'autre part, $J_{R}^{(3)}$ est défini par :

$$
\begin{aligned}
J_{R}^{(3)}=\frac{\rho^{2}}{V} \iiint & {\left[3\left(\mathbf{r}_{p s} \cdot \mathbf{r}_{s q}\right)^{2}-r_{p s}^{2} \cdot r_{s q}^{2}\right] \times } \\
& \times p_{p s}^{-5} r_{s q}^{-5} g\left(\mathbf{r}_{p} \cdot \mathbf{r}_{q} \cdot \mathbf{r}_{s}\right) \mathrm{d} \mathbf{r}_{p} \mathrm{~d} \mathbf{r}_{q} \mathrm{~d} \mathbf{r}_{s}
\end{aligned}
$$

$J_{R}^{(3)}$ est le paramètre de corrélations radiales ternaires [27] dans lequel intervient la fonction de corrélation ternaire $g\left(\mathbf{r}_{p}, \mathbf{r}_{q}, \mathbf{r}_{s}\right)$ couplant trois molécules situées au même instant aux points $\mathbf{r}_{p}, \mathbf{r}_{q}$ et $\mathbf{r}_{\boldsymbol{s}}$.

Le paramètre de corrélation binaire [48] est accessible au calcul grâce aux modèles de Kirkwood [32] et de Lennard-Jones généralisé [46]. De même, on peut calculer le paramètre de corrélation ternaire [49] par des méthodes utilisées dans le traitement d'autres phénomènes [43], [45], [46]. Ce paramètre n'a jamais, à notre connaissance, été pris jusqu'à ce jour en considération dans les calculs numériques. Il paraît jouer un rôle important, puisque son apport peut être positif dans certains modèles et négatif dans d'autres. On s'en persuadera en considérant deux cas extrêmes : pour des vecteurs $\mathbf{r}_{p s}$ et $\mathbf{r}_{s q}$ presque parallèles (structure asymétrique), $J_{R}^{(3)}$ est positif et contribue à augmenter l'anisotropie; d'autre part, si les vecteurs $\mathbf{r}_{p s}$ et $\mathbf{r}_{s q}$ sont quasi perpendiculaires (structure symétrique), le paramètre $J_{R}^{(3)}$ devient négatif et abaisse l'anisotropie effective (47). Cette dernière hypothèse paraît être la plus probable car au fur et à mesure que la densité augmente, la structure de l'entourage immédiat d'une molécule devient de plus en plus symétrique. Les corrélations multiples entrent alors en jeu et peuvent entraîner un abaissement de l'anisotropie effective. On peut penser que les collisions ternaires (et d'ordres plus élevés) jouent également un rôle important dans le spectre de la lumière dépolarisée diffusée par les gaz, phénomène dont une étude expérimentale détaillée vient d'être donnée [47].

c) Application aux molécules de Langevin. - En substituant, dans la formule (41) du déviateur, le champ moléculaire exprimé par l'équation (45), on obtient :

$$
\begin{aligned}
D_{i j}^{(p)}=a_{i j}^{(p)}-a_{p} \delta_{i j}-\frac{1}{2} \sum_{s \neq p}^{N}\left(a_{i k}^{(p)} a_{l j}^{(s)}+a_{j k}^{(p)} a_{l i}^{(s)}\right) T_{k l}^{(p s)}+ & \\
& +\frac{1}{3} \delta_{i j} \sum_{s \neq p}^{N} a_{k l}^{(p)} T_{l m}^{(p s)} a_{m k}^{(s)}
\end{aligned}
$$

Pour les molécules de Langevin d'anisotropie $\gamma=a_{33}-a_{11}$, nous avons, d'après $(16 b)$ :

$$
a_{i j}^{(p)}=a_{p} \delta_{i j}+\gamma_{p}\left(k_{i}^{(p)} k_{j}^{(p)}-\frac{1}{3} \delta_{i j}\right)
$$

$\mathbf{k}^{(p)}$ désigne le vecteur-unité dans la direction de l'axe de symétrie (axe principal 3) de la molécule $p$. En introduisant les expressions (50) et (51) dans l'anisotropie effective (14), on obtient, en permière approximation et en ne tenant compte que des corrélations binaires :

$$
\begin{aligned}
\Gamma_{\lambda}^{2}=\gamma_{\lambda}^{2}\left(1+J_{A}\right)+4 & \gamma_{\lambda}\left\{\left[3 a_{\lambda}\left(a_{\lambda}-\frac{1}{3} \gamma_{\lambda}\right)\right] J_{R A}^{(1)}+\right. \\
& \left.+2 \gamma_{\lambda}\left(a_{\lambda}+\frac{1}{3} \gamma_{\lambda}\right) J_{R A}^{(2)}\right\}
\end{aligned}
$$

le paramètre de corrélation angulaire $J_{A}$ est donné par la formule (24). On constate en considérant la formule (52) que de nouveaux paramètres mixtes redistribution-corrélations angulaires sont apparus, notamment :

$$
\begin{array}{r}
J_{R A}^{(1)}=\frac{1}{4} Z<\left(3 \cos ^{2} \theta_{p}+3 \cos ^{2} \theta_{q}-2\right) r_{p q}^{-3}> \\
J_{R A}^{(2)}=\frac{1}{2} Z<\left(3 \cos \theta_{p q} \cdot \cos \theta_{p} \cdot \cos \theta_{q}-\right. \\
\left.-\cos ^{2} \theta_{p q}\right) r_{p q}^{-3}>.
\end{array}
$$

Les angles $\theta_{p}$ et $\theta_{q}$ sont ceux que les axes de symétrie $\mathbf{k}_{p}$ et $\mathbf{k}_{q}$ font avec le vecteur $\mathbf{r}_{p q}$. Ces paramètres résultent d'un couplage entre le processus de corrélations angulaires et celui de fluctuations de translation (ou de redistribution moléculaire). En l'absence de corrélations angulaires, la moyenne sur toutes les orientations possibles annule ces termes croisés. Dans le cas général, les calculs de ces paramètres mixtes présentent des difficultés. On peut cependant les effectuer en admettant, quand elle est fondée, l'hypothèse simplificatrice des corrélations faibles [7], [48]. Dans le cas des corrélations fortes étudiées, nous préférerons évaluer ces termes à partir de modèles très simples décrits par la suite. Notons cependant que dans ce dernier cas, nous pouvons éliminer d'une manière satisfaisante la dépendance explicite des paramètres (53) et (54) avec $r_{p q}^{-3}$, en ayant recours à la relation (25). II vient alors :

$$
\begin{aligned}
J_{R A}^{(1)}= & \frac{Z \rho}{4 g}\left\{3<\cos ^{2} \theta_{p}>\right. \\
J_{R A}^{(2)}= & \frac{Z \rho}{2 g}\left\{3<\cos ^{2} \theta_{q}>-2\right\} \\
- & \left.<\cos ^{2} \theta_{p q}>\right\}
\end{aligned}
$$

Dans le benzène, pour une configuration à tendance perpendiculaire, on a :

$$
\cos \theta_{p}=1, \quad \cos \theta_{q}=\cos \theta_{p q},
$$

d'où :

$$
\begin{aligned}
& J_{R A}^{(1)}=\frac{\rho}{2 g}\left(Z+J_{A}\right), \\
& J_{R A}^{(2)}=\frac{\rho}{3 g}\left(Z+2 J_{A}\right),
\end{aligned}
$$

$J_{A}$, dans le cas des corrélations fortes, est donné par (24a). Pour une telle configuration, l'effet mixte décrit fournit un apport négatif à l'anisotropie effective, 
compte tenu de l'anisotropie négative du benzène, du nombre de proches voisins $Z$ supérieur ou égal à 1 et de la valeur du paramètre. $J_{A}$ comprise entre $-0,5$ et 0 .

Dans l'éventualité d'une structure moléculaire du benzène à tendance parallèle, on a

$$
\cos \theta_{p}=\cos \theta_{q}=0, \quad \cos \theta_{p q} \neq 0,
$$

et les paramètres $(53 a),(54 a)$ se réduisent à :

$$
\begin{aligned}
J_{R A}^{(1)} & =-\frac{Z \rho}{2 g}, \\
J_{R A}^{(2)} & =-\frac{\rho}{6 g}\left(Z+2 J_{A}\right) .
\end{aligned}
$$

On constate que, dans cette configuration, l'apport de l'effet mixte est positif compte tenu de la valeur de $J_{A}$ comprise entre 0 et +1 . L'anisotropie effective est alors augmentée.

Des effets similaires ont été étudiés par Takatsuji [49] et récemment par Buckingham et al. [50] dans le cas des solutions très diluées.

Quoi qu'il en soit, ces calculs négligent l'apport des corrélations ternaires et d'ordre plus élevé. Leurs contributions pourront être soit positives soit négatives et nous ne sommes pas en mesure de déterminer actuellement pour les molécules que nous avons étudiées, le signe exact du paramètre rendant compte de l'effet mixte redistribution-corrélations angulaires.

D. Techniques de mesure du paramètre $J_{A}$ et de ses variations thermiques dans le cas des molécules fortement anisotropes. - 1. LA DIFFUSION RAYLEIGH DÉPOLARISÉE. - Elle nous permet de déterminer la valeur de $J_{A}$ à température ordinaire. Nous avons utilisé de nombreux appareillages utilisant les lasers, continus ou déclenchés décrits dans des publications antérieures [8]. Nous mesurons directement la composante dépolarisée (éq. 1) convenablement détectée puis filtrée. L'information obtenue est proportionnelle à $\int_{\omega} i(\omega) \mathrm{d} \omega$ où $i(\omega)$ est l'intensité dépolarisée diffusée à la fréquence $\omega$ dans la bande $\mathrm{d} \omega$. La bande large passante des filtres utilisés (environ $300 \mathrm{~cm}^{-1}$ à $\lambda=546,1 \mathrm{~nm}$ ) intègre les informations provenant de mécanismes initiateurs cer, ainement variés et encore bien mal connus.

Le signal obtenu est proportionnel à l'anisotropie optique apparente précédemment définie (éq. 14). Le calcul du paramètre $J_{A}$ (éq. 24) s'effectue en :

1) négligeant la contribution due à la redistribution moléculaire (éq. 47). Nous avons calculé l'ordre de grandeur de ce paramètre, dans le cas du benzène et du nitrobenzène, en supposant les corrélations binaires prépondérantes : le rapport calculé $\frac{9_{\alpha}^{-4} J_{R}}{\gamma^{2}}$ est, dans ces deux cas, inférieur à 0,05 et notre approximation semble très légitime [51] ;

2) évaluant l'influence du champ interne $F$ en utilisant le modèle d'Onsager qui se prête particulièrement bien aux calculs numériques [5 5 ]. Le calcul du terme correctif $\left(1+A_{\text {ani }}\right)$ suppose la connaissance des polarisabilités optiques moléculaires de la molécule isolée et de l'indice de réfraction du milieu ;

3) comparant les anisotropies apparentes de la molécule, au sein du liquide pur et dans une solution très diluée (extrapolation à concentration nulle) effectuée au moyen d'un solvant constitué de molécules très isotropes (cyclohexane) [8].

2. L'efFet KeRr OPTIQUe. - Il nous permet la détermination des variations thermiques du paramètre $J_{A}$. Les détails expérimentaux de nos montages ont été récemment publiés [15], [17]. Nous mesurons directement la constante $B\left(\lambda_{I}, \lambda_{A}\right)$ définie par l'équation (6), à plusieurs températures situées dans l'intervalle température de fusion-température d'ébullition. La mesure de la constante $B$, comme celle de l'intensité $i$, conduisent directement à la valeur de l'anisotropie optique moléculaire apparente définie, éq. 14.

La connaissance de la variation thermique du paramètre $A_{\text {ani }}$ aisément calculable, autorise alors le calcul des variations thermiques de $J_{A}$.

Remarque : L'une quelconque des deux techniques citées suffit à la mesure de $J_{A}$ et de ses variations thermiques. Notre choix (Diffusion Rayleigh Dépolarisée pour la mesure de $J_{A}$ et Effet Kerr Optique pour la détermination de ses $_{q}$ variations thermiques) est uniquement dicté par des considérations techniques.

E. Technique de mesure du paramètre $J_{R}$. - L'hyperpolarisabilité condamne l'effet Kerr optique [20]. Nous utilisons uniquement la diffusion Rayleigh dépolarisée. La mesure de $\Gamma_{\lambda}^{2}$ et l'utilisation de l'équation (47) conduisent à la détermination de $J_{R}$.

F. Résultats et discussion. - 1. CORRÉlations ANGULAIREs. - Nous avons étudié le benzène et le nitrobenzène. Le tableau I précise les éléments essentiels nécessaires à la détermination du paramètre $J_{A}$ et nos calculs effectués à partir des mesures de diffusion Rayleigh dépolarisée.

Il faut noter que la connaissance des fluctuations anisotropes du champ interne et des corrélations d'orientation permet le calcul de l'anisotropie de la molécule «isolée », par exemple à partir de l'anisotropie apparente de la molécule dans le liquide, au moyen de l'équation [8], [52] :

$$
\gamma_{0}^{2}=\frac{\gamma_{\text {iq }}^{2}}{\left(1+A_{\mathrm{ani}}\right)\left(1+J_{A}\right)} .
$$

Les anisotropies ainsi calculées sont très voisines des valeurs mesurées en phase vapeur (tableau I).

Nous avons également mesuré les variations thermiques du paramètre $J_{A}$ dans l'intervalle $5-85^{\circ} \mathrm{C}$. Les tableaux II et III précisent les valeurs obtenues et la figure 3 illustre ces résultats.

a) Dans le cas du nitrobenzène, la valeur positive, voisine de +1 , du paramètre $J_{A}$, traduit une orienta- 


\section{TABLE I}

Mesure du paramètre $J_{A}$ par diffusion Rayleigh dépolarisée

Température $21^{\circ} \mathrm{C}$; longueur d'onde : $546,1 \mathrm{~nm}$

\begin{tabular}{|c|c|c|c|c|c|c|c|c|}
\hline $\begin{array}{l}\text { Com- } \\
\text { posé }\end{array}$ & $\begin{array}{c}\Gamma_{\text {liq }}^{2}\left(\AA^{6}\right) \\
\text { liquide } \\
\text { pur } \\
\text { mesure } \\
\text { DRD }\end{array}$ & $\begin{array}{c}\Gamma^{2} \text { sol }\left(\AA^{6}\right) \\
\text { Solution } \\
\text { cyclohexane } \\
\text { extrapolation } \\
\text { à concentration } \\
\text { nulle }\end{array}$ & $\begin{array}{c}\left(1+A_{\mathrm{ani}}\right) \\
\text { liquide } \\
\text { pur }\end{array}$ & $\begin{array}{c}\left(1+A_{\mathrm{ani}}\right) \\
\text { solution } \\
\text { cyclohexane } \\
\text { extrapolation } \\
\text { à concentration } \\
\text { nulle }\end{array}$ & $\begin{array}{c}J_{A} \\
\text { nos } \\
\text { valeurs }\end{array}$ & $\begin{array}{c}J_{A} \\
\text { autres } \\
\text { auteurs }\end{array}$ & $\begin{array}{c}\text { Anisotropie } \\
\text { optique de la } \\
\text { molécule isolée } \\
\text { au moyen } \\
\text { de éq. } 55\end{array}$ & $\begin{array}{l}\text { Anisotropie } \\
\text { optique } \\
\text { mesurée } \\
\text { dans } \\
\text { la vapeur }\end{array}$ \\
\hline Benzène & $30, \overline{5}(t)$ & $\overline{38,7 \pm 0,4}$ & $\overline{1,26}$ & $\overline{1,27}$ & $-\overline{0,21}$ & $\begin{array}{l}-\overline{-} \\
-0,18[57] \\
-0,24[8] \\
-0,19[58]\end{array}$ & $\overline{30,6}$ & $31,7\left(^{*}\right)$ \\
\hline $\begin{array}{l}\text { Nitro- } \\
\text { benzène }\end{array}$ & $201 \pm 4$ & $95 \pm 4$ & 1,40 & 1,41 & $+1,13$ & $\begin{array}{l}1,25[57] \\
1,20[11] \\
1,27[58]\end{array}$ & 67,4 & $74,9(\$)$ \\
\hline
\end{tabular}

(†) Valeur calculée à partir de $R$ benzène $=16,3 \times 10^{-6} \mathrm{~cm}^{-1} ; D_{v}=0,42$.

$\left({ }^{*}\right)$ Valeur calculée à partir de $D_{v}=0,038$; référence : (A.), MAssoulier C. R. Acad. Sci. Paris, 1960, $358,251$.

(§) Références : StuaRT (H. A.), Die Struktur des freien Moleküls, Springer-Verlag Berlin 1952, tables 98 et 99.

\section{TABLE II}

Variations thermiques des corrélations d'orientation dans le benzène. Les valeurs expérimentales du paramètre $J_{A}$ sont obtenues par effet Kerr optique. La valeur † est calculée en diffusion Rayleigh dépolarisée à partir de $R=16,3 \times 10^{-6} \mathrm{~cm}^{-1}$ et $D_{v}=0,42$.

$\begin{array}{lccccccccc}\text { Température }\left({ }^{\circ} \mathrm{C}\right) & 5 & 5,2 & 6,4 & 10,3 & 15 & 20,4 & 21 & 55,5 & 69 \\ A_{\text {ani }} & 0,227 & 0,277 & 0,276 & 0,274 & 0,271 & 0,268 & 0,255 & 0,237 & 0,230 \\ J_{A} & -0,57 & -0,35 & -0,31 & -0,26 & -0,24 & -0,22 & -0,21 & -0,10 & -0,01 \\ \gamma_{T}^{2} \text { en } \AA^{6} & 17 \pm 3 & 25 \pm 3 & 27 \pm 4 & 29 \pm 5 & 30 \pm 5 & 30 \pm 5 & 30,5(\dagger) & 34 \pm 5 & 38 \pm 6\end{array}$

\section{TABLE III}

Variations thermiques des corrélations d'orientation dans le nitrobenzène. Les valeurs expérimentales du paramètre $J_{A}$ sont obtenues par effet Kerr optique. La valeur $\dagger$ a été mesurée par diffusion Rayleigh dépolarisée.

Température

$\begin{array}{lccccccccc}\left.{ }^{\circ} \mathrm{C}\right) & 5,2 & 5,9 & 10 & 15,3 & 21 & 41,2 & 55,5 & 70 & 84 \\ A_{\text {ani }} & 0,398 & 0,398 & 0,395 & 0,392 & 0,388 & 0,377 & 0,369 & 0,361 & 0,354 \\ \gamma_{T}^{2} \text { en } \AA^{\prime} & 206 \pm 30 & 208 \pm 30 & 199 \pm 30 & 203 \pm 30 & 201 \pm 4(\dagger) & 199 \pm 30 & 197 \pm 30 & 189 \pm 30 & 185 \pm 30 \\ \boldsymbol{J}_{\boldsymbol{A}} & +1,17 & +1,19 & +1,10 & +1,14 & +1,13 & +1,13 & +1,12 & +1,04 & +1,01\end{array}$

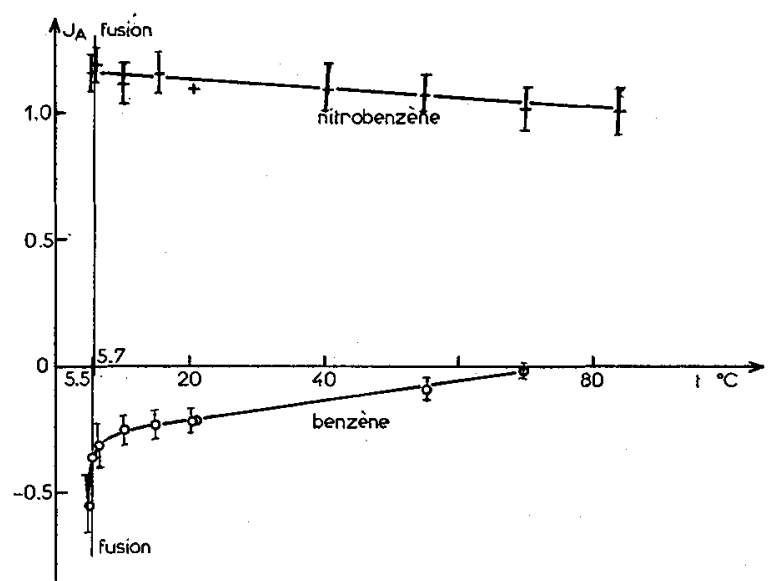

FIG. 3. -- Variations thermiques du paramètre $J_{A}$, dans les cas du benzène et du nitrobenzène. tion moyenne de type parallele (paratropisme [53]). Nous ne remarquons aucune variation de $J_{A}$ dans le voisinage immédiat de la fusion. Il semble que l'ordre orientationnel, existant au sein du monocristal, subsiste dans le liquide et ne diminue que très lentement quand la température s'élève.

Les corrélations d'orientation dans le solide semblent devoir se prolonger au-dessus du point de fusion. Ce comportement est à rapprocher de celui mis récemment en évidence [54] dans les cristaux de type «plastique » [55]: prolongement de l'aspect optique de la dynamique orientationnelle du liquide au-dessous du point de fusion.

b) Le benzène présente un comportement très différent. Les molécules, comme dans le monocristal, présentent une orientation moyenne de type perpendi- 
culaire (diatropisme [53]) traduite par la valeur négative du paramètre $J_{A}$. Il faut noter le bon accord de ce résultat avec des données récentes de diffraction $X$ [56]. L'orientation diminue spectaculairement au voisinage de la fusion puis décroît lentement et régulièrement pour s'annuler près de l'ébullition.

2. Corrélations radiales. - La mesure directe de la composante dépolarisée diffusée permet la détermination de $J_{R}$ dans le cas de liquides constitués de molécules isotropes. Nous avons ainsi mesuré le paramètre $J_{R}$ dans le cas du tétrachlorure de carbone, en utilisant la relation :

$$
H_{V}=\frac{16}{15}\left(\frac{\pi}{\lambda}\right)^{4}\left(\frac{n^{2}+2}{3}\right)^{2} \frac{N}{V} 9 a^{4} J_{R} I
$$

obtenue en portant (47) dans (14) puis dans (2) et (1).

Nous avons obtenu la valeur :

$$
J_{R}=(144 \pm 3) \times 10^{+41} \mathrm{~cm}^{-6}
$$

en bon accord avec celle donnée en référence [33] $\left(J_{R}=130 \times 10^{41} \mathrm{~cm}^{-6}\right)$ mais très inférieure à la valeur théorique calculée grâce au modèle de Kirkwood [32] $\left(J_{R}=3,2 \times 10^{44} \mathrm{~cm}^{-6}\right)$.

G. Conclusion. - $\mathrm{Ce}$ travail met en évidence le comportement très différent de deux liquides, benzène et nitrobenzène, quant aux corrélations d'orientation existant entre leurs molécules. Cet ordre "orientationnel » présente les caractéristiques suivantes :

- dans le cas du benzène : dû principalement à l'interaction de tendance perpendiculaire (quadrupôlequadrupôle, quadrupôle-hexadécapôle...), il est de type diatropique et semble diminuer très rapidement au voisinage de la fusion;

- dans le cas du nitrobenzène : dû principalement à l'interaction antiparallèle (dipôle-dipôle, dipôleoctupôle...), il est de type paratropique et semble persister sans diminution notable après la fusion.

Ces résultats expérimentaux sont en assez bon accord avec nos calculs théoriques, basés sur des modèles très simples et que nous devons maintenant améliorer. Ces modèles, d'ores et déjà, conduisent à des signes corrects du paramètre $J_{A}$ et à des variations thermiques calculées raisonnables.

Ils doivent permettre la détermination de certains moments électriques moléculaires d'ordre élevé encore très mal connus dans les liquides : moment quadrupolaire par exemple.

Ce travail illustre, de plus, l'importance de deux phénomènes trop souvent négligés dans les études de diffusion Rayleigh dépolarisée :

- la redistribution due aux fluctuations de translation des molécules dont l'apport à l'anisotropie effective est toujours positif dans l'approximation des corrélations binaires. Mais les termes d'ordre supérieur peuvent largement contribuer à une diminution de cet apport.

- l'effet croisé redistribution-corrélations angulaires pouvant, toujours dans l'approximation des corrélations binaires, soit augmenter, soit diminuer l'anisotropie optique effective.

La prise en considération de l'ensemble de ces phénomènes permet d'obtenir - à notre connaissance pour la première fois - un accord très satisfaisant entre les valeurs de l'anisotropie optique, mesurées dans la vapeur, -dans le liquide et dans la solution très diluée faite à partir d'un solvant isotrope.

Remerciements. - Nous sommes heureux de remercier le Professeur P. Bothorel avec qui nous avons souvent discuté certains points de ce travail.

H. Appendice. - Calculs de l'énergie potentielle d'orientation dans quelques liquides.

L'énergie potentielle totale d'interaction des molécules du milieu est donnée par :

$$
W\left(\tau^{N}\right)=\frac{1}{2} \sum_{p=1}^{N} \sum_{q=1}^{N} W_{p q}\left(\tau_{p}, \tau_{q}\right)
$$

où $W_{p q}\left(\tau_{p}, \tau_{q}\right)$ est l'énergie d'interaction binaire entre molécules $p$ et $q$ présentant les configurations $\tau_{p}$ et $\tau_{q}$.

Dans le cas où les molécules ne possèdent pas de moments électriques permanents, la partie due à l'orientation des forces de dispersion anisotropes de London est de la forme [35] :

$$
\begin{aligned}
W_{p q}^{\mathrm{dis}-\mathrm{ani}}= & -\frac{1}{4} \frac{h v_{p} h v_{q}}{h v_{p}+h v_{q}} \\
& \times\left(\alpha_{\alpha \beta}^{(p)} \alpha_{\alpha \beta}^{(q)}-\alpha_{p} \alpha_{q} \delta_{\alpha \beta} \delta_{\gamma \delta}\right) T_{\alpha y}^{(p q)} T_{\beta \dot{\delta}}^{(p q)},
\end{aligned}
$$

où $h v_{p}$ et $h v_{q}$ sont deux énergies caractéristiques des molécules $p$ et $q$.

Si le tenseur de polarisabilité électrique est exprimé dans le référentiel principal de la molécule $p$, on a :

$$
\begin{aligned}
\alpha_{\alpha \beta}^{(p)}=\alpha_{p} & \delta_{\alpha \beta}+\frac{1}{2}\left(\alpha_{11}^{(p)}-\alpha_{22}^{(p)}\right)\left(i_{\alpha}^{(p)} i_{\beta}^{(p)}-j_{\alpha}^{(p)} j_{\beta}^{(p)}\right)+ \\
& +\frac{1}{2}\left(2 \alpha_{33}^{(p)}-\alpha_{11}^{(p)}-\alpha_{22}^{(p)}\right)\left(k_{\alpha}^{(p)} k_{\beta}^{(p)}-\frac{1}{3} \delta_{\alpha \beta}\right)
\end{aligned}
$$

où

$$
i_{\alpha}=c_{\alpha_{1}}, j_{\alpha}=c_{\alpha_{2}}, k_{z}=c_{\alpha_{3}} .
$$

Pour des molécules de Langevin (3) se réduit à :

$$
\alpha_{\alpha \beta}^{(p)}=\alpha_{p} \delta_{\alpha \beta}+\gamma_{p}\left(k_{\alpha}^{(p)} k_{\beta}^{(p)}-\frac{1}{3} \delta_{\alpha \beta}\right)
$$

où $\gamma_{p}=\alpha_{33}^{(p)}-\alpha_{11}^{(p)}$ et $\mathbf{k}^{(p)}$ est le vecteur porté par l'axe de symétrie de la molécule $p$. 
En introduisant (4) dans (2), on obtient :

$$
\begin{aligned}
W_{p q}^{\mathrm{dis}-\mathrm{ani}}=\frac{1}{4} \frac{h v_{p} h v_{q}}{h v_{p}+h v_{q}}\left\{\alpha_{p} \gamma_{q}+\gamma_{p} \alpha_{q}-\gamma_{p}\left(3 \alpha_{q}-\gamma_{q}\right) \cos ^{2} \theta_{p}-\gamma_{q}\left(3 \alpha_{p}-\gamma_{p}\right) \cos ^{2} \theta_{q}-\right. \\
\left.-\gamma_{p} \gamma_{q}\left(3 \cos \theta_{p} \cos \theta_{q}-\cos \theta_{p q}\right)^{2}\right\} r_{p q}^{-6},
\end{aligned}
$$

où $\theta_{p}$ et $\theta_{q}$ sont les angles faits par les axes de symétrie $\mathbf{k}_{p}$ et $\mathbf{k}_{q}$ des deux molécules et le vecteur $\mathbf{r}_{p q}$ et $\theta_{p q}$ l'angle des vecteurs $\mathbf{k}_{p}$ et $\mathbf{k}_{q}$.

1. Benzène. - Pour ce liquide constitué de molécules centro-symétriques, on peut définir les contributions suivantes :

a) Interaction quadrupôle-quadrupôle

$$
W_{p q}^{\Theta-\Theta}=-\frac{1}{9} \Theta_{\alpha \beta}^{(p)} T_{\alpha \beta \gamma \delta}^{(p q)} \Theta_{\gamma \delta}^{(q)},
$$

où $\Theta_{\alpha \beta}$ est le tenseur décrivant le moment quadrupolaire et $T_{\alpha \beta \gamma \delta}^{(p q)}$ le tenseur décrivant les interactions quadrupôlequadrupôle.

b) Interactions quadrupôle-hexadécapôle [35]

$$
W_{p q}^{\Theta-\Phi}=-\frac{1}{315}\left\{\Theta_{\alpha \beta}^{(p)} \Phi_{\gamma \delta \varepsilon \eta}^{(q)}+\Phi_{\alpha \beta \gamma \delta}^{(p)} \Theta_{\varepsilon \eta}^{(q)}\right\} T_{\alpha \beta \gamma \delta \varepsilon \eta}^{(p q)},
$$

$\Phi_{\alpha \beta \gamma \delta}$ est le tenseur décrivant le moment hexadécapolaire et $T_{\alpha \beta \gamma \delta \varepsilon \eta}$ le tenseur décrivant les interactions du type quadrupôle-hexadécapôle.

c) Interaction dipôle induit-quadrupôle [35] :

$$
W_{p q}^{\alpha-\Theta}=-\frac{1}{18}\left\{\alpha_{\alpha \beta}^{(p)} \Theta_{\gamma \delta}^{(q)} \Theta_{\varepsilon \eta}^{(q)}+\alpha_{\alpha \beta}^{(q)} \Theta_{\gamma \delta}^{(p)} \Theta_{\varepsilon \eta}^{(p)}\right\} T_{\alpha \gamma \delta}^{(p q)} T_{\beta \varepsilon \eta}^{(p q)}
$$

$T_{\alpha \gamma \delta}$ est le tenseur décrivant les interactions dipôle-quadrupôle.

Pour les molécules de Langevin (et pour les groupes ponctuels $C_{3 h}, C_{2 h}, C_{6 v}$ ) nous avons, en plus de l'équation (4) :

$$
\begin{aligned}
\Theta_{\alpha \beta}^{(p)}= & \frac{1}{2} \Theta_{p}\left(3 k_{\alpha} k_{\beta}-\delta_{\alpha \beta}\right), \\
\Phi_{\alpha \beta \gamma \delta}^{(p)}= & \frac{1}{8} \Phi_{p}\left\{35 k_{\alpha}^{(p)} k_{\beta}^{(p)} k_{\gamma}^{(p)} k_{\delta}^{(p)}-5\left(k_{\alpha}^{(p)} k_{\beta}^{(p)} \delta_{\gamma \delta}+k_{\beta}^{(p)} k_{\gamma}^{(p)} \delta_{\delta \alpha}+k_{\gamma}^{(p)} k_{\delta}^{(p)} \delta_{\alpha \beta}+k_{\alpha}^{(p)} k_{\gamma}^{(p)} \delta_{\beta \delta}+\right.\right. \\
& \left.\left.+k_{\alpha}^{(p)} k_{\delta}^{(p)} \delta_{\beta \gamma}+k_{\beta}^{(p)} k_{\delta}^{(p)} \delta_{\alpha \gamma}\right)+\delta_{\alpha \beta} \delta_{\gamma \delta}+\delta_{\alpha \gamma} \delta_{\beta \delta}+\delta_{\alpha \delta} \delta_{\beta \gamma}\right\}
\end{aligned}
$$

et les expressions (6), (7) et (8) se réduisent à :

$$
\begin{aligned}
W_{p q}^{\Theta-\Theta}= & \frac{p^{3}}{4} \Theta_{p}^{\top} \Theta_{q}\left\{1-5\left(\cos ^{2} \theta_{p}^{\prime}+3 \cos ^{2} \theta_{p} \cos ^{2} \theta_{q}+\cos ^{2} \theta_{q}\right)+2\left(5 \cos \theta_{p} \cos \theta_{q}-\cos \theta_{p q}\right)^{2}\right\} r_{p q}^{-5}, \\
W_{p q}= & \frac{15}{16}\left\{\Theta _ { p } \Phi _ { q } \left[231 \cos ^{2} \theta_{p} \cos ^{4} \theta_{q}-21\left(\cos ^{4} \theta_{q}+6 \cos ^{2} \theta_{p} \cos ^{2} \theta_{q}+8 \cos \theta_{p} \cos ^{3} \theta_{q} \cos \theta_{p q}\right)+\right.\right. \\
& \left.+7\left(\cos ^{2} \theta_{p}+2 \cos ^{2} \theta_{q}+4 \cos ^{2} \theta_{q} \cos ^{2} \theta_{p q}+8 \cos \theta_{p} \cos \theta_{q} \cos \theta_{p q}\right)-4 \cos ^{2} \theta_{p q}-1\right] \\
& +\Phi_{p} \Theta_{q}\left[231 \cos ^{2} \theta_{q} \cos ^{4} \theta_{p}-21\left(\cos ^{4} \theta_{p}+6 \cos ^{2} \theta_{q} \cos ^{2} \theta_{p}+8 \cos \theta_{q} \cos ^{3} \theta_{p} \cos \theta_{p q}\right)\right. \\
& \left.\left.+7\left(\cos ^{2} \theta_{q}+2 \cos ^{2} \theta_{p}+4 \cos ^{2} \theta_{p} \cos ^{2} \theta_{p q}+8 \cos \theta_{p} \cos \theta_{q} \cos \theta_{p q}\right)-4 \cos ^{2} \theta_{p q}-1\right]\right\} r_{p q}^{-7} . \\
W_{p q}^{\alpha-\Theta}=- & \frac{3}{8}\left\{\left(3 \alpha_{p}-\gamma_{p}\right) \Theta_{q}^{2}\left(1-2 \cos ^{2} \theta_{q}+5 \cos ^{4} \theta_{q}\right)+\Theta_{p}^{2}\left(3 \alpha_{q}-\gamma_{q}\right)\left(1-2 \cos ^{2} \theta_{p}+5 \cos ^{4} \theta_{q}\right)+\right. \\
& +3 \gamma_{p} \Theta_{q}^{2}\left(5 \cos \theta_{p} \cos ^{2} \theta_{q}-2 \cos \theta_{p q} \cos \theta_{q}-\cos \theta_{p}\right)^{2} \\
& \left.+3 \gamma_{q} \Theta_{p}^{2}\left(5 \cos \theta_{q} \cos ^{2} \theta_{p}-2 \cos \theta_{p q} \cos \theta_{p}-\cos \theta_{q}\right)^{2}\right\} r_{p q}^{-8} .
\end{aligned}
$$

Dans le cas du benzène et pour une tendance d'orientation de type perpendiculaire :

$$
\cos \theta_{p}=1, \quad \cos \theta_{q}=\cos \theta_{p q}
$$


(on retrouvera aisément le modèle [53] en faisant $\theta_{p q}=\pi / 2$ ). Les expressions (5), (9)-(11) se simplifient et s'écrivent :

$$
\begin{aligned}
& W_{p q}^{\mathrm{dis}-\mathrm{ani}}=-\frac{1}{8} \gamma h v\left\{2 \alpha+(\alpha+\gamma)\left(3 \cos ^{2} \theta_{p q}-1\right)\right\} r_{p q}^{-6}, \\
& W_{p q}^{\Theta-\Theta}=3 \Theta^{2}\left(3 \cos ^{2} \theta_{p q}-1\right) r_{p q}^{-5}, \\
& W_{p q}^{\Theta-\Phi}=-\frac{15}{8} \Theta \Phi\left(1+18 \cos ^{2} \theta_{p q}-35 \cos ^{4} \theta_{p q}\right) r_{p q}^{-7}, \\
& W_{p q}^{\alpha-\Theta}=-\frac{3}{8} \Theta^{2}\left\{3(5 \alpha-2 \gamma)-2(3 \alpha-4 \gamma) \cos ^{2} \theta_{p q}+(15 \alpha+22 \gamma) \cos ^{4} \theta_{p q}\right\} r_{p q}^{-8} .
\end{aligned}
$$

La relation (25) permet de mettre ces résultats sous la forme (26).

2. NrTRoBenZÈNE. - Pour les molécules non centro-symétriques, on définit les contributions suivantes :

a) Interaction dipôle-dipôle

$$
W_{p q}^{\mu-\mu}=\mu_{\alpha}^{(p)} T_{\alpha \beta}^{(p q)} \mu_{\beta}^{(q)}
$$

$\mu_{\alpha}^{(p)}$ et $\mu_{\beta}^{(q)}$ sont les moments dipolaire électrique des molécules $p$ et $q$.

b) Interaction dipôle-quadrupôle

$$
W_{p q}^{\mu-\theta}=\frac{1}{3}\left\{\mu_{\alpha}^{(p)} \Theta_{\beta \gamma}^{(q)}-\Theta_{\alpha \beta}^{(p)} \mu_{\gamma}^{(q)}\right\} T_{\alpha \beta \gamma}^{(p q)} .
$$

c) Interaction dipôle-octopôle

$$
W_{p q}^{\mu-\Omega}=\frac{1}{15}\left\{\mu_{\alpha}^{(p)} \Omega_{\beta \alpha \delta}^{(q)}+\Omega_{\alpha \beta \gamma}^{(p)} \mu_{\delta}^{(q)}\right\} T_{\alpha \beta \gamma \delta}^{(p q)},
$$

d) Interaction quadrupôle-octopôle [35]

$$
W_{p q}^{\Theta-\Omega}=\frac{1}{45}\left\{\Theta_{\alpha \beta}^{(p)} \Omega_{\gamma \delta \varepsilon}^{(q)}-\Omega_{\alpha \beta \gamma}^{(p)} \Theta_{\delta \varepsilon}^{(q)}\right\} T_{\alpha \beta \gamma \delta \varepsilon}^{(p q)},
$$

e) Interaction octopôle-octopôle

$$
W_{p q}^{\Omega-\Omega}=\frac{1}{225} \Omega_{\alpha \beta \gamma}^{(p)} T_{\alpha \beta \gamma \delta \varepsilon \eta}^{(p q)} \Omega_{\delta \varepsilon \eta}^{(q)},
$$

f) Interaction dipôle induit-dipôle permanent

$$
W_{p q}^{\alpha-\mu}=-\frac{1}{2}\left\{\alpha_{\alpha \beta}^{(p)} \mu_{\gamma}^{(q)} \mu_{\delta}^{(q)}+\alpha_{\alpha \beta}^{(q)} \mu_{\gamma}^{(p)} \mu_{\delta}^{(p)}\right\} T_{\alpha \gamma}^{(p q)} T_{\beta \delta}^{(p q)} .
$$

Pour les molécules appartenant au groupe $C_{2 v}$ (p. ex. $\mathrm{H}_{2} \mathrm{O}, \mathrm{C}_{6} \mathrm{H}_{5} \mathrm{NO}_{2}$ ) les composantes des tenseurs multipolaires électriques s'écrivent :

$$
\begin{aligned}
\mu_{\alpha}^{(p)}= & \mu_{3}^{(p)} \\
\Theta_{\alpha}^{(p)} & \\
\Theta_{\alpha \beta}^{(p)}= & \frac{1}{2}\left(\Theta_{11}^{(p)}-\Theta_{22}^{(p)}\right)\left(i_{\alpha}^{(p)} i_{\beta}^{(p)}-j_{\alpha}^{(p)} j_{\beta}^{(p)}\right)+\frac{1}{2} \Theta_{33}^{(p)}\left(3 k_{\alpha}^{(p)} k_{\beta}^{(p)}-\delta_{\alpha \beta}\right) \\
\Omega_{\alpha \beta \gamma}^{(p)}= & \frac{1}{2}\left(\Omega_{113}^{(p)}-\Omega_{223}^{(p)}\right) \sum_{3}\left(i_{\alpha}^{(p)} i_{\beta}^{(p)}-j_{\alpha}^{(p)} j_{\beta}^{(p)}\right) k_{\gamma}^{(p)}+ \\
& +\frac{1}{2} \Omega_{333}^{(p)}\left(5 k_{\alpha}^{(p)} k_{\beta}^{(p)} k_{\gamma}^{(p)}-\sum_{3} \delta_{\alpha \beta} k_{\gamma}^{(p)}\right), \\
\Phi_{\alpha \beta \gamma \delta}^{(p)}=\frac{1}{2}( & \left.\Phi_{1133}^{(p)}-\Phi_{2233}^{(p)}\right)\left[7\left(j_{\alpha}^{(p)} j_{\beta}^{(p)} j_{\gamma}^{(p)} j_{\delta}^{(p)}-i_{\alpha}^{(p)} i_{\beta}^{(p)} i_{\gamma}^{(p)} i_{\delta}^{(p)}\right)+\sum_{6}\left(i_{\alpha}^{(p)} i_{\beta}^{(p)}-j_{\alpha}^{(p)} j_{\beta}^{(p)}\right) \delta_{\gamma \delta}\right]+ \\
& +\frac{1}{8}\left(\Phi_{1133}^{(p)}+8 \Phi_{1122}^{(p)}+\Phi_{2233}^{(p)}\right)\left[3 k_{\alpha}^{(p)} k_{\beta}^{(p)} k_{\gamma}^{(p)} k_{\delta}^{(p)}\right. \\
& \left.\quad 4\left(i_{\alpha}^{(p)} i_{\beta}^{(p)} i_{\gamma}^{(p)} i_{\delta}^{(p)}+j_{\alpha}^{(p)} j_{\beta}^{(p)} j_{\gamma}^{(p)} j_{\delta}^{(p)}\right)-\sum_{\sigma} k_{\alpha}^{(p)} k_{\beta}^{(p)} \delta_{\gamma \delta}+\sum_{3} \delta_{\alpha \beta} \delta_{\gamma \delta}\right] \\
& +\frac{1}{8} \Phi_{3333}^{(p)}\left(35 k_{\alpha}^{(p)} k_{\beta}^{(p)} k_{\gamma}^{(p)} k_{\delta}^{(p)}-5 \sum_{6} k_{\alpha}^{(p)} k_{\beta}^{(p)} \delta_{\gamma \delta}+\sum_{3} \delta_{\alpha \beta} \delta_{\gamma \delta}\right),
\end{aligned}
$$


où $\sum_{3}$ et $\sum_{6}$ définissent des sommes comprenant 3 et 6 termes obtenus par permutation des indices correspondant dans l'expression écrite.

Dans le cas de molécules ayant la symétrie axiale (groupe $C_{\infty o v}$ ) on aura en outre :

$$
\Theta_{11}=\Theta_{22}, \quad \Omega_{113}=\Omega_{223}, \quad \Phi_{1133}=\Phi_{2233}=-4 \Phi_{1122}
$$

et les énergies (12)-(27) deviennent [35] :

$$
\begin{aligned}
& W_{p q}^{\mu-\mu}=-\mu_{p} \mu_{q}\left(3 \cos \theta_{p} \cos \theta_{q}-\cos \theta_{p q}\right) r_{p q}^{-3}, \\
& W_{p q}^{\mu-\Theta}=\frac{3}{2}\left\{\mu_{p} \Theta_{q}\left(5 \cos \theta_{p} \cos ^{2} \theta_{q}-2 \cos \theta_{p q} \cos \theta_{q}-\cos \theta_{p}\right)-\right. \\
& \left.-\Theta_{p} \mu_{q}\left(5 \cos \theta_{q} \cos ^{2} \theta_{p}-2 \cos \theta_{p q} \cos \theta_{p}-\cos \theta_{q}\right)\right\} r_{p q}^{-4} \text {, } \\
& W_{p q}^{\mu-\Omega}=-\frac{1}{2}\left\{\mu_{p} \Omega_{q}\left(35 \cos \theta_{p} \cos ^{3} \theta_{q}-15 \cos \theta_{p} \cos \theta_{q}-15 \cos \theta_{p} \cos \theta_{q} \cos \theta_{p q}+3 \cos \theta_{p q}\right)\right. \\
& \left.+\Omega_{p} \mu_{q}\left(35 \cos ^{3} \theta_{p} \cos \theta_{q}-15 \cos \theta_{p} \cos \theta_{q}-15 \cos \theta_{p} \cos \theta_{q} \cos \theta_{p q}+3 \cos \theta_{p q}\right)\right\} r_{p q}^{-5} \text {, } \\
& W_{p q}^{\Theta-\Omega}=\frac{5}{4}\left\{\Theta _ { p } \Omega _ { q } \left(63 \cos ^{2} \theta_{p} \cos ^{3} \theta_{q}-42 \cos \theta_{p} \cos ^{2} \theta_{q} \cos \theta_{p q}-\right.\right. \\
& \left.-21 \cos ^{2} \theta_{p} \cos \theta_{q}+6 \cos \theta_{q} \cos ^{2} \theta_{p q}-7 \cos ^{3} \theta_{q}+6 \cos \theta_{p} \cos \theta_{p q}+3 \cos \theta_{q}\right) \\
& -\Omega_{p} \Theta_{q}\left(63 \cos ^{3} \theta_{p} \cos ^{2} \theta_{q}-42 \cos \theta_{q} \cos ^{2} \theta_{p} \cos \theta_{p q}-21 \cos ^{2} \theta_{q} \cos \theta_{p}+6 \cos \theta_{p} \cos ^{2} \theta_{p q}\right. \\
& \left.\left.-7 \cos ^{3} \theta_{p}+6 \cos \theta_{q} \cos \theta_{p q}+3 \cos \theta_{p}\right)\right\} r_{p q}^{-6} \text {, } \\
& W_{p q}^{\Omega-\Omega}=-\frac{5}{4} \Omega_{p} \Omega_{q}\left\{21\left(11 \cos ^{2} \theta_{p} \cos ^{2} \theta_{q}-3 \cos ^{2} \theta_{p}-3 \cos ^{2} \theta_{q}-6 \cos \theta_{p} \cos \theta_{q} \cos \theta_{p q}\right) \cos \theta_{p} \cos \theta_{q}\right. \\
& \left.+\left(21 \cos ^{2} \theta_{p}+2 \cos ^{2} \theta_{q}-2\right) \cos \theta_{p q}+\left(21 \cos \theta_{p} \cos \theta_{q}-\cos \theta_{p q}\right)\left(1+2 \cos ^{2} \theta_{p q}\right)\right\} r_{p q}^{-7} \text {, } \\
& W_{p q}^{\alpha-\mu}=-\frac{1}{6}\left\{\left(3 \alpha_{p}-\gamma_{p}\right) \mu_{q}^{2}\left(3 \cos ^{2} \theta_{q}+1\right)+\mu_{p}^{2}\left(3 \alpha_{q}-\gamma_{q}\right)\left(3 \cos ^{2} \theta_{p}+1\right)\right. \\
& \left.+3\left(\gamma_{p} \mu_{q}^{2}+\mu_{p}^{2} \gamma_{q}\right)\left(3 \cos \theta_{p} \cos \theta_{q}-\cos \theta_{p q}\right)^{2}\right\} r_{p q}^{-6} \text {. }
\end{aligned}
$$

Pour le nitrobenzène qui présente une tendance à l'orientation de type antiparallèle :

$$
\cos \theta_{p}=\cos \theta_{q}=0, \quad \cos \left(\pi-\theta_{p q}\right)=-\cos \theta_{p q}
$$

et les expressions (5), (9), (10), (11), (12a)-(17a) deviennent :

$$
\begin{aligned}
W_{p q}^{\mu-\mu} & =-\mu^{2} r_{p q}^{-3} \cos \theta_{p q}, \\
W_{p q}^{\mu-\Theta} & =0, \\
W_{p q}^{\mu-\Omega} & =3 \mu \Omega r_{p q}^{-5} \cos \theta_{p q}, \\
W_{p q}^{\Theta-\Theta} & =\frac{3}{4} \Theta^{2} r_{p q}^{-5}\left(1+2 \cos ^{2} \theta_{p q}\right), \\
W_{p q}^{\Theta-\Omega} & =0 \\
W_{p q}^{\Omega-\Omega} & =-\frac{15}{4} \Omega^{2} r_{p q}^{-7}\left(\cos \theta_{p q}+\frac{2}{3} \cos ^{3} \theta_{p q}\right), \\
W_{p q}^{\Theta-\Phi} & =-\frac{15}{8} \Theta \Phi r_{p q}^{-7}\left(1+4 \cos ^{2} \theta_{p q}\right), \\
W_{\gamma q}^{\mathrm{dis}-\mathrm{ani}} & =\frac{1}{8} \gamma h v r_{p q}^{-6}\left(2 \alpha-\gamma \cos ^{2} \theta_{p q}\right), \\
W_{p q}^{\alpha-\mu} & =-\frac{1}{3} \mu^{2}\left(3 \alpha-\gamma+3 \gamma \cos ^{2} \theta_{p q}\right) r_{p q}^{-6}, \\
W_{p q}^{\alpha-\theta} & =-\frac{3}{4}(3 \alpha-\gamma) \Theta^{2} r_{p q}^{-8} .
\end{aligned}
$$

La relation (25) permet de mettre ces résultats sous la forme (31). 


\section{Bibliographie}

[1] Dezelic (G.), J. Chem. Phys., 1966, 45, 185 ; Dezelic (G.) et Vavra (J.), Croat. Chem. Acta, 1966, 38,35 .

[2] Kielich (S.), J. Chem. Phys., 1967, 46, 4090.

[3] SicotTe (Y.), J. Chem. Phys., 1967, 64, 584.

[4] Bothorel (P.), J. Colloid Interf. Sci., 1968, 27, 529.

[5] SCHMidt (R. L.), ibid., 1968, 27, 516.

[6] Malmberg (M.S.) et LipPincotT, ibid., 1968, 27, 591.

[7] Kielich (S.), J. Physique, 1968, 29, 619; Acta Phys. Polonica, 1968, 33, 65 .

[8] Lalanne (J. R.), J. Physique, 1969, 30, 643.

[9] Ghita (C.), Revue Roumanie de Chimie, 1968, 13, 1019 ; Shakmparonov (M. I.), Vestnik Moskovskovo Universiteta, Khimia, 1969, 3, 3.

[10] Coumou (D. J.), Trans. Faraday Soc., 1969, 65, 2654.

[11] Fechner (B. M.), Acta Phys. Polonica, 1969, 36, 297.

[12] Such (Ch.), Clément (C.) et Bothorel (P.), $C . R$. Acad. Sci. Paris, 1970, 271C, 228.

[13] Kielich (S.) et Pieczynska (J.), Bull. Soc. Amis. Sci. Lettres Poznan, 1970, 22B, in press.

[14] Hellwarth (R. W.), J. Chem. Phys., 1970, 52, 2128.

[15] Martin (F. B.) et LalanNe (J. R.), Optics Communications, 1970, 2, 219.

[16] Champion (J. V.), Meeten (G. H.) et Whittle (Ch. D.), J. Chim. Phys., 1970, 67, 1684.

[17] Martin (F. B.) et Lalanne (J. R.), Phys. Rev., 1971.

[18] Kielich (S.), Proc. Phys. Soc., 1967, 90, 847.

[19] Meeten (G. H.), Trans. Faraday Soc., 1968, 64, 1776.

[20] Kielich (S.), LalanNe (J. R.) et Martin (F. B.), C. R. Acad. Sci. Paris, 1971, 272B, 731.

BOTHOREL (P.), Ann. chim., 1959, 669 ;

Piaud (J. J.), J. Chim. Phys., 1962, 215.

[21] Lalanne (J. R.) et Bothorel (P.), J. Chim. Phys., $1966,11 \mathrm{13}, 1538$.

[22] Kielich (S.), Acta Phys. Polonica, 1960, 19, 149, 573.

[23] Mayer (G.) et Gires (F.), C. R. Acad. Sci. Paris, 1964, 258, 2039.

[24] Paillette (M.), Ann. Phys. Paris, 1969, 4, 671.

[25] Kasprowicz (B.) et Kielich (S.), Acta Phys. Polonica, $1968,33,495$.

[26] Volgt (W.), Ann. Phys., 1901, 4, 197.

[27] Kielich (S.), Acta Phys. Polonica, 1966, 30, 683.

[28] Kielich (S.) et Piekara (A.), Acta Phys. Polonica, $1959,18,439$.

[29] BORN (M.), Optic/J. Springer, Berlin, 1933.

[30] Langevin (P.), Le Radium, 1910, 7, 249.

[31] Yvon (J.), C. R. Acad. Sci. Paris, 1936, 202, 35.

[32] Kirkwood (J. G.), J. Chem. Phys., 1936, 4, 592.

[33] Kielich (S.), Acta Phys. Polonica, 1962, 22, 299.
[34] Kielich (S.), Chem. Phys. Letters, 1968, 2, 112

[35] Kielich (S.), Acta Phys. Polonica, 1962, 22, 65 ; 1964, $25,39$.

[36] Benoit (H.) et Stockmayer (W. H.), J. Phys. Radium, $1956,17,21$.

[37] Kielich (S.), Bull. Acad. Polon. Sci. Ser. Sci. Math. Astron. Phys., 1958, 6, 215.

[38] Peterlin (A.) et Stuart (H. A.), Z. Phys., 1939, 113, 663.

[39] Pibkara (A.), Acta Phys. Polonica, 1950, 10, 107 ;

Piekara (A.) et Kielich (S.), J. Physique Radium, $1957,18,490$.

[40] Kielich (S.), Acta Phys. Polonica, 1958, 17, 239.

[41] Stuart (H. A.), Molekülstruktur/Springer Verlag, Berlin, 1967.

[42] KieLICH (S.), Opto-electronics, 1970, 2, 5 ; Acta Phys. Polonica, 1970, 37A, 447, 719.

[43] Hellwarth. (R. W.), Phys. Rev., 1966, 152, 56 ; Quantum Optics. Edited by R. J. Glauber, Academic Press, New York, 1969, p. 563.

[44] Kielich (S.), Chem. Phys. Letters, 1970, 7, 347.

[45] Kielich (S.), Acta Phys. Polonica, 1965, 27, 395, 913 ; 28, 95,459 .

[46] Stogryn (D. E.), J. Chem. Phys., 1969, 50, 4967.

[47] Thibeau (M.), Tabisz (G. C.), OKsengorn (B.) et VODAR (B.), J. Q. S. R. T., 1970, 10, 839.

McTague (J. P.) et Birnbaum (G.), Phys. Rev., 1971, 3A, 1376.

148] Krelich (S.), Acta Phys. Polonica, 1960, 19, 711.

[49] Takatsuri (M.), Phys. Rev., 1968, 165, 171.

[50] Buckingham (A. D.), Stiles (P. J.) et RITCHIE (G. L. D.), Trans. Faraday Soc., 1971, 67, 577.

[51] Lalanne (J. R.), Bothorel (P.) et Martin (F. B.), à paraître dans The Journal of colloid and Interface Science, 1971.

[52] Kielich (S.), Acta Phys. Polonica, 1961, 20, 83 ; Poz. Soc. Fr. Sci., Math and Nat. Sci., 1962, 11, 3.

[53] Prins (J. A.) et Prins (W.), Physica, 1957, 23, 253.

[54] JaCkson (D. A.), Bird (M. J.), Pentecost (H. T. A.) et Powles (J. G.), Phys. Letters, 1971, 35A, 1.

[55] Timmermans (J.), Bull. Soc. Chim. Belg., 1935, 44, 17.

[56] Narten (A. H.), J. Chem. Phys., 1968, 48, 1630; Mikusinska (A.), Bull. Soc. Amis Sci. Lettres, Poznan, 1968-69, 21B, 77.

[57] Kielich (S.) et Surma (M.), Poz. Soc. Fr. Sci. Math. and Nat. Sci. 1962, 11, 153 ;

Surma (M.), Acta Phys. Polonica, 1964, 25, 485.

[58] LuCAS (H. C.) et JACKSON (D. A.), Molecular Physics, $1971,20,801$. 